November 1992

SUSX-TH-92/17.

\title{
Twisted Sector Yukawa Couplings For The $\mathbf{Z}_{M} \times \mathbf{Z}_{N}$ Orbifolds
}

\author{
D. BAILin $\lambda a$, A. Love $\lambda b$ AND W.A. SABRA $\lambda c^{\star}$

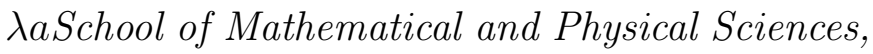 \\ University of Sussex, \\ Brighton U.K. \\ $\lambda b$ Department of Physics, \\ Royal Holloway and Bedford New College, \\ University of London, \\ Egham, Surrey, U.K. \\ $\lambda c$ Physics Department \\ Birkbeck College, \\ University of London, \\ Malet Street \\ London WC1E rHX
}

\begin{abstract}
The moduli dependent Yukawa couplings between twisted sectors of $\mathbf{Z}_{M} \times \mathbf{Z}_{N}$ Coxeter orbifolds are studied.

\footnotetext{
$\star$ Address from January 1993, Department of Physics, Royal Holloway and Bedford New College, University of London, Surrey, U.K.
} 


\section{Introduction}

A knowledge of the Yukawa couplings for orbifold compactified string theory models $[1,2]$ is an important step towards comparison of the models with observation. Of particular importance is the exponential dependence of Yukawa couplings on moduli $[3,4]$ which can arise when all the states involved are in twisted sectors, because of its possible bearing on hierarchies [5]. Twisted sector Yukawa coulping have been investigated for the $\mathbf{Z}_{3}$ orbifold [3-6], for the $\mathbf{Z}_{7}$ orbifold [7] and for arbitrary $\mathbf{Z}_{N}$ orbifolds [8-11]. Here, we shall extend our recent discussion [12] of Yukawa couplings for the $\mathbf{Z}_{3} \times \mathbf{Z}_{3}$ orbifold to all Coxeter $\mathbf{Z}_{M} \times \mathbf{Z}_{N}$ orbifolds with the point group realized by Coxeter elements acting on a direct sum of twodimensional root lattices. After completion of the present work we have received a preprint by Stieberger [13] which also addresses the question of Yukawa couplings for $\mathbf{Z}_{M} \times \mathbf{Z}_{N}$ orbifolds though with the emphasis on general properties such as modular symmetries rather than specific orbifolds on specific lattices. A possible advantage that $\mathbf{Z}_{M} \times \mathbf{Z}_{N}$ orbifolds possess over $\mathbf{Z}_{N}$ orbifolds is that they can more generically provide the string loop threshold corrections needed to explain the low energy values of the gauge coupling constants [14-18] because they are not so tightly constrained by duality anomaly cancellation conditions [17].

\section{Yukawa couplings for $\mathbf{Z}_{M} \times \mathbf{Z}_{N}$ Orbifolds.}

To specify an orbifold model it is necessary to specify both the point group and the lattice on which the point group acts as a symmetry. Perhaps the most elegant possibility [19] is that the point group acts as a Coxeter element (product of Weyl reflections) on a root lattice of a Lie algebra. Here, we shall study the case where the root lattice involved is a direct sum of three 2-dimensional root lattices. For the $\mathbf{Z}_{M} \times \mathbf{Z}_{N}$ orbifolds (table 1) the factors involved are $\mathbf{Z}_{3}, \mathbf{Z}_{4}, \mathbf{Z}_{6}$ and $\mathbf{Z}_{2}$. These discrete groups can be realised as the Coxeter elements for the $S U(3), S O(5), G_{2}$ and $S O(4)$ root lattices, respectively $[20,19]$. The $\mathbf{Z}_{2}$ group can also be realized as the square of the Coxeter element of the $S O(5)$ lattice and the cube of the Coxeter 
element on the $G_{2}$ lattice, and the $\mathbf{Z}_{3}$ group can also be realized as the square of the Coxeter element on the $G_{2}$ lattice. The simplest possibility (table 1 of the present work, and table 1 of ref. 20) is to realize $\mathbf{Z}_{3}, \mathbf{Z}_{4}$ and $\mathbf{Z}_{6}$ as a Coxeter element on each of the two dimensional sub-lattices (rather than as a power of Coxeter element). This can be done for each of the $\mathbf{Z}_{M} \times \mathbf{Z}_{N}$ orbifolds, except $\mathbf{Z}_{3} \times \mathbf{Z}_{6}$, by the choice of lattices in table 1 , and for $\mathbf{Z}_{3} \times \mathbf{Z}_{6}$ it can be done for 2 of the 3 sub-lattices with $\mathbf{Z}_{3}$ realized as the square of the Coxeter element on the third $G_{2}$ sub-lattice. These are the $\mathbf{Z}_{M} \times \mathbf{Z}_{N}$ models we shall study here.

The action of the Coxeter element $C(G)$ of a rank 2 Lie algebra $G$ on the basis vectors $e_{1}$ and $e_{2}$ of the root lattices may be taken to be

$$
\begin{gathered}
C(S U(3)) e_{1}=e_{2}, \quad C(S U(3)) e_{2}=-e_{1}-e_{2}, \\
C(S O(5)) e_{1}=e_{1}+2 e_{2}, \quad C(S O(5)) e_{2}=-e_{1}-e_{2}, \\
C\left(G_{2}\right) e_{1}=-e_{1}-e_{2}, \quad C\left(G_{2}\right) e_{2}=3 e_{1}+2 e_{2},
\end{gathered}
$$

and

$$
C(S O(4)) e_{1}=-e_{1}, \quad C(S O(4)) e_{2}=-e_{2}
$$

realizing $\mathbf{Z}_{3}, \mathbf{Z}_{4}, \mathbf{Z}_{6}$ and $\mathbf{Z}_{2}$, respectively. The powers of Coxeter elements $C \lambda 2(S O(5))$ and $C \lambda 3\left(G_{2}\right)$ realizing $\mathbf{Z}_{2}$ have the same action as $C(S O(4))$, and $C \lambda 2\left(G_{2}\right)$ realizing $\mathbf{Z}_{3}$ has the action

$$
C \lambda 2\left(G_{2}\right) e_{1}=-2 e_{1}-e_{2}, \quad C \lambda 2\left(G_{2}\right) e_{2}=3 e_{1}+e_{2} .
$$

The non-zero Yukawa couplings obey point group selection rules, and selection rules for the H-lattice momentum associated with bosonized right-moving NSR fermionic degrees of freedom. These selection rules have already been written down [20] for $\mathbf{Z}_{M} \times \mathbf{Z}_{N}$ orbifolds. They also obey space group selection rules [3,4] 
which may be written in the following form. Let $\left(\alpha, l_{1}\right),\left(\beta, l_{2}\right)$ and $\left(\gamma, l_{3}\right)$ be space group elements associated with the $\alpha, \beta$ and $\gamma$ twisted sectors, where

$$
\begin{aligned}
& l_{1}=(I-\alpha) f_{\alpha}+(I-\alpha) \Lambda, \\
& l_{2}=(I-\beta) f_{\beta}+(I-\beta) \Lambda,
\end{aligned}
$$

and

$$
l_{3}=(I-\gamma) f_{\gamma}+(I-\gamma) \Lambda
$$

In (2.7)-(2.8), $f_{\alpha}, f_{\beta}$ and $f_{\gamma}$ are fixed points or fixed tori for the $\alpha, \beta$ and $\gamma$ twisted sectors, respectively, and in each case $\Lambda$ denotes an arbitrary lattice vector. Then, given that we have already satisfied the point group selection rule

$$
\alpha \beta \gamma=I
$$

the full space group selection rule contains the additional condition

$$
l_{1}+l_{2}+l_{3} \text { contains } 0 \text {. }
$$

The values of the non-zero Yukawa couplings amongst the twisted sectors are determined in detail by three-point functions involving fermionic and bosonic degrees of freedom. However, the crucial dependence on the deformation parameters (moduli) and the particular fixed points and fixed tori is entirely contained in (bosonic) twist field correlation functions $[3,4]$ of the type

$$
\mathcal{Z}=\prod_{i=1} \lambda 3<\sigma_{\alpha} \lambda i\left(z_{1}, \bar{z}_{1}\right) \sigma_{\beta} \lambda i\left(z_{2}, \bar{z}_{2}\right) \sigma_{\gamma} \lambda i\left(z_{3}, \bar{z}_{3}\right)>=\prod_{i=1} \lambda 3 \mathcal{Z}_{i}
$$

where $\alpha, \beta$ and $\gamma$ now label the twisted sectors and also the fixed points or fixed tori involved, and the index $i$ distinguishes the twist fields associated with the complex 
coordinates $X_{i}, i=1,2,3$, that define the six-dimensional compact manifold. The correlation function

$$
\mathcal{Z}_{i}=<\sigma_{\alpha} \lambda i\left(z_{1}, \bar{z}_{1}\right) \sigma_{\beta} \lambda i\left(z_{2}, \bar{z}_{2}\right) \sigma_{\gamma} \lambda i\left(z_{3}, \bar{z}_{3}\right)>
$$

factors $[3,4]$ into a quantum piece $\mathcal{Z} \lambda i_{q u}$ and a classical piece with all the dependence on the moduli and the particular fixed points and fixed tori involved contained in the classical piece.

$$
\mathcal{Z}_{i}=\mathcal{Z} \lambda i_{q u} \sum_{X_{c l}} e \lambda-S \lambda i_{c l}
$$

where the classical action is

$$
S \lambda i_{c l}=\frac{1}{\pi} \int d \lambda 2 z\left(\frac{\partial X_{i}}{\partial z} \frac{\partial \bar{X}_{i}}{\partial \bar{z}}+\frac{\partial X_{i}}{\partial \bar{z}} \frac{\partial \bar{X}_{i}}{\partial z}\right) .
$$

Because of the string equations of motion

$$
\frac{\partial \lambda 2 X_{i}}{\partial z \partial \bar{z}}=0
$$

$\partial X_{i} / \partial z$ and $\partial X_{i} / \partial \bar{z}$ are functions of $z$ and $\bar{z}$ alone, respectively, which have to be chosen to respect the boundary conditions at $z_{1}, z_{2}$ and $z_{3}$ implicit in the operator product expansions with the twist fields [3]. Let the space group elements for the fixed points or fixed tori involved in the Yukawa coupling be $\left(\alpha, l_{1}\right),\left(\beta, l_{2}\right)$ and $\left(\gamma, l_{3}\right)$, as before, with $l_{1}, l_{2}$ and $l_{3}$ as in (2.6)-(2.8). Also let $P$ be the least common denominator for the fractional twists involved so that the action of the point group elements $\alpha, \beta$ and $\gamma$ on the three complex coordinates $X_{i}$ may be written in the form

$$
\begin{gathered}
\alpha: X_{i} \rightarrow e \lambda 2 \pi i A_{i} / P X_{i}, \\
\beta: X_{i} \rightarrow e \lambda 2 \pi i B_{i} / P X_{i}, \\
\gamma: X_{i} \rightarrow e \lambda 2 \pi i C_{i} / P X_{i},
\end{gathered}
$$


where $A_{i}, B_{i}$ and $C_{i}$ are positive integers smaller than $P$ with

$$
A_{i}+B_{i}+C_{i}=0(\bmod P)
$$

for consistency with the point group selection rule. When one of the $A_{i}, B_{i}$ or $C_{i}$ is zero, that is one of the $\alpha, \beta$ or $\gamma$ leaves the $i$-th complex plane invariant, then the three twist field correlation function reduces to a 2 twist field correlation function, which may be normalized to one. Otherwise, we need to evaluate the classical action using

$$
\frac{\partial X_{i}}{\partial z}=a_{i}\left(z-z_{1}\right) \lambda-\left(1-A_{i} / P\right)\left(z-z_{2}\right) \lambda-\left(1-B_{i} / P\right)\left(z-z_{3}\right) \lambda-\left(1-C_{i} / P\right)
$$

and

$$
\frac{\partial X_{i}}{\partial \bar{z}}=b_{i}\left(\bar{z}-\bar{z}_{1}\right) \lambda-A_{i} / P\left(\bar{z}-\bar{z}_{2}\right) \lambda-B_{i} / P\left(\bar{z}-\bar{z}_{3}\right) \lambda-C_{i} / P .
$$

In all the cases we shall study here, only the holomorphic field $\partial X_{i} / \partial z$ is an acceptable classical solution, because $\partial X_{i} / \partial \bar{z}$ gives a divergent contribution to the classical action and we must set

$$
b_{i}=0
$$

The constants $a_{i}$ may be evaluated by the use of global monodromy conditions [3] where we integrate around a closed contour $\mathcal{C}_{i}$ such that $X_{i}$ is shifted by $v_{i}$ but not rotated. Thus,

$$
\oint_{\mathcal{C}_{i}} d z \frac{\partial X_{i}}{\partial z}=v_{i}
$$

where we have dropped the contribution of $\partial X_{i} / \partial \bar{z}$ as just discussed. If, for example, we choose $\mathcal{C}_{i}$ to go $B_{i}$ times around $z_{1}$ counter-clockwise and $A_{i}$ times around $z_{2}$ clockwise, with the choice (allowed by $S L(2, C)$ symmetry)

$$
z_{1}=0, \quad z_{2}=1, \quad z_{3}=\infty
$$

then $X_{i}$ is not rotated. Multiplying together the relevant space group elements, $\left(\alpha, l_{1}\right) \lambda B_{i}\left(\beta \lambda-1, l_{2}\right) \lambda A_{i}$ we find the shift in $i$-th complex plane to be the projection 
into this plane of

$$
W_{i}=\left(1-\alpha \lambda B_{i}\right)\left(f_{\alpha}-f_{\beta}+\Lambda\right),
$$

where $\Lambda$ is an arbitrary lattice vector. In the first instance, (2.23) is in the lattice basis for the Coxeter orbifold. If we transform to the orthonormal basis in which the action of $\alpha, \beta$ and $\gamma$ is given by (2.16), then $v_{i}$ is the component of $W_{i}$ in the $i$-th complex direction, e.g. for $X_{1}$ the shift $v_{1}$ is the component of $W_{1}$ in the $1+i 2$ direction, where 1 and 2 denote the orthonormal basis. The global monodromy condition may be evaluated with the aid of the integral

$$
\oint_{\mathcal{C}_{i}} d z z \lambda-\left(1-A_{i} y\right)(z-1) \lambda-\left(1-B_{i} y\right)=-2 i \sin \left(A_{i} B_{i} \pi y\right) \frac{\Gamma\left(A_{i} y\right) \Gamma\left(B_{i} y\right)}{\Gamma\left(A_{i} y+B_{i} y\right)}
$$

with the result

$$
2 i a_{i} \sin \left(\frac{A_{i} B_{i} \pi}{P}\right) \frac{\Gamma\left(\frac{A_{i}}{P}\right) \Gamma\left(\frac{B_{i}}{P}\right)}{\Gamma\left(\frac{A_{i}}{P}+\frac{B_{i}}{P}\right)}\left(-z_{\infty}\right) \lambda-\left(1-C_{i} / P\right)=v_{i}
$$

which determines $a_{i}$. The contribution to the classical action may now be obtained by using Appendix A of [21], with the result

$$
S_{c l} \lambda i=\frac{\left|v_{i}\right| \lambda 2}{4 \pi \sin \lambda 2\left(\frac{A_{i} B_{i} \pi}{P}\right)} \frac{\sin \left(\frac{A_{i} \pi}{P}\right) \sin \left(\frac{B_{i} \pi}{P}\right)}{\sin \left(\frac{\left(A_{i}+B_{i}\right) \pi}{P}\right)} .
$$

Exactly similar expressions may be written down when the contour is chosen instead to encircle the pair of twist fields associated with $\left(\alpha, l_{1}\right)$ and $\left(\gamma, l_{3}\right)$, or the pair of twist fields associated with $\left(\beta, l_{2}\right)$ and $\left(\gamma, l_{3}\right)$. In general, to obtain consistency between these different global monodromy conditions it is necessary in (2.13) to restrict the sum over the initially arbitrary lattice vectors arising in $v_{i}$ in a way which depends on the fixed tori involved. This problem has been solved for the case of $\mathbf{Z}_{N}$ orbifolds in appendix $\mathrm{B}$ of the last reference of [11], and this same result can be applied here provided we restrict attention to one particular complex coordinate $X_{i}$ at a time. 
In general, after general GSO projections inclusive of Wilson lines [22, 2] the surviving physical states are eigenstates of the two point group elements $\theta$ and $\omega$ generating the $\mathbf{Z}_{M} \times \mathbf{Z}_{N}$ point group. In the construction of these $\theta$ and $\omega$ eigenstates account must be taken of the fact that fixed points (or tori) are not always left invariant [23, 9] by the action of $\theta$ or $\omega$. If $m$ and $n$ are the least integers such that for the fixed point (or torus) $f_{k l}$ of $\theta \lambda k \omega \lambda l$,

$$
\theta \lambda m f_{k l} \sim f_{k l}
$$

and

$$
\omega \lambda n f_{k l} \sim f_{k l}
$$

where $\sim$ signifies up to a lattice vector, then the $\theta$ and $\omega$ eigenstate combinations of fixed points $\mid p, q>$ are of the form

$\left|p, q>=\sum_{r=0} \lambda m-1 \sum_{s=0} \lambda n-1 e \lambda-i 2 \pi p r / m e \lambda-i 2 \pi q s / n\right| \theta \lambda r \omega \lambda s f_{k l}>, \quad p=0, . ., m-1 \quad q=0, . ., n-1$

which are simultaneous eigenstates of $\theta$ and $\omega$ with eigenvalues $e \lambda i 2 \pi p / m$ and $e \lambda i 2 \pi q / n$, respectively.

Then, the Yukawa couplings for physical states are obtained as combinations of terms for definite fixed tori, subject to the space group selection rules. 


\section{The $\mathbf{Z}_{2} \times \mathbf{Z}_{6}$ orbifold}

In this section, we shall illustrate the calculations and results by considering the case of the $\mathbf{Z}_{2} \times \mathbf{Z}_{6}$ Coxeter orbifold on the $S O(4) \times G_{2} \lambda 2$ lattice as in table 1 . The corresponding results for the other $\mathbf{Z}_{M} \times \mathbf{Z}_{N}$ orbifolds are given in summary in the appendices. In the orthonormal basis, the action of the generators $\theta$ and $\omega$ of the point group on the complex string degrees of freedom $X_{i}, i=1,2,3$, is

$$
\theta X_{1}=-X_{1}, \quad \theta X_{2}=X_{2}, \quad \theta X_{3}=-X_{3},
$$

and

$$
\omega X_{1}=X_{1}, \quad \omega X_{2}=e \lambda 2 \pi i / 6 X_{2}, \quad \omega X_{3}=e \lambda-2 \pi i / 6 X_{3} .
$$

On the other hand, for this Coxeter orbifold, the action of $\theta$ and $\omega$ on the lattice basis is given by

$$
\theta=\left(C(S O(4)), I, C \lambda-3\left(G_{2}\right)\right)
$$

and

$$
\omega=\left(I, C\left(G_{2}\right), C \lambda-1\left(G_{2}\right)\right),
$$

where $C(H)$ is the Coxeter element for the Lie algebra of $H$. Explicitly, using (2.3) and (2.4), the action on the 6 basis vectors $e_{1}, \ldots, e_{6}$ for the lattice basis is

$$
\begin{aligned}
& \theta e_{1}=-e_{1}, \quad \theta e_{3}=e_{3}, \quad \theta e_{5}=-e_{5}, \\
& \theta e_{2}=-e_{2}{ }^{\prime} \quad \theta e_{4}=e_{4}{ }^{\prime} \quad \theta e_{6}=-e_{6}
\end{aligned}
$$

and

$$
\begin{aligned}
& \omega e_{1}=e_{1}, \quad \omega e_{3}=-e_{3}-e_{4}, \quad \omega e_{5}=2 e_{5}+e_{6}, \\
& \omega e_{2}=e_{2} \quad \omega e_{4}=3 e_{3}+2 e_{4}, \quad \omega e_{6}=-3 e_{5}-e_{6} \text {. }
\end{aligned}
$$

More generally, the original rigid lattice may be deformed in ways which preserve the action (3.5) and (3.6) of the point group. To obtain the most general choice of 
lattice compatible with the point group we must require that all the scalar products $e_{i} . e_{j}$ are preserved by the transformations (3.5) and (3.6). If we write

$$
e_{i} \cdot e_{j}=\left|e_{i}\right|\left|e_{j}\right| \cos \theta_{i j}, \quad i \neq j
$$

we find that

$$
\begin{aligned}
& \left|e_{4}\right|=\sqrt{3}\left|e_{3}\right| \equiv \sqrt{3} R_{3}, \\
& \left|e_{6}\right|=\sqrt{3}\left|e_{5}\right| \equiv \sqrt{3} R_{5},
\end{aligned}
$$

and

$$
\cos \theta_{34}=\cos \theta_{56}=-\frac{\sqrt{3}}{2}
$$

with all other $\cos \theta_{i j}$ zero except $\cos \theta_{12}$, and

$$
\begin{aligned}
& \left|e_{1}\right| \equiv R_{1}, \\
& \left|e_{2}\right| \equiv R_{2},
\end{aligned}
$$

and $\cos \theta_{12}$ undetermined. Thus we may take 5 independent deformations of the lattice compatible with the point group to be $R_{1}, R_{2}, R_{3}, R_{5}$ and $\cos \theta_{12}$. These are the deformation parameters or moduli. The fixed points and fixed tori for the twisted sectors are readily obtained using the action (3.5) and (3.6) of the generators of the point group on the (deformed) lattice. These are presented in table 2 , for the twisted sectors containing massless states together with the associated space group elements. The space group selection rules for the various Yukawa couplings amongst twisted sectors are then easily obtained from (2.10). All Yukawa couplings consistent with the point group selection rule and H-momentum conservation rule for the bosonized NSR fermion right movers have already been listed in [20]. As already discussed in section 2, the physical states surviving generalized GSO projections are $\theta$ and $\omega$ eigenstates, which particular eigenstates survive 
depending on the details of the point group embeddings and Wilson lines in a particular model. The $\theta$ and $\omega$ eigenstate combinations of fixed points (or tori) may be obtained from the action of $\theta$ and $\omega$ on the fixed tori given in table 3. An orthonormal basis $\tilde{e}_{1}, \ldots, \tilde{e}_{6}$ in which $X_{1}, X_{2}$ and $X_{3}$ are the components of the string degrees of freedom in the $1+i 2,2+i 3$ and $5+i 6$ directions, respectively, is obtained by comparing (3.1) and (3.2) with (3.5) and (3.6), and using (3.8)-(3.10) when ensuring the orthonormality of the basis. A suitable choice is related to the lattice basis by

$$
\begin{aligned}
& e_{1}=R_{1} \tilde{e}_{1}, \quad e_{3}=R_{3} \tilde{e}_{3}, \quad e_{5}=R_{5} \tilde{e}_{5}, \\
& e_{2}=R_{2}\left(\cos \theta_{12} \tilde{e}_{1}+\sin \theta_{12} \tilde{e}_{2}\right), \quad e_{4}=-R_{3}\left(\frac{3}{2} \tilde{e}_{3}+\frac{\sqrt{3}}{2} \tilde{e}_{4}\right), \quad e_{6}=-R_{5}\left(\frac{3}{2} \tilde{e}_{5}+\frac{\sqrt{3}}{2} \tilde{e}_{6}\right)
\end{aligned}
$$

The moduli and fixed point (or tori) dependence of the non-zero Yukawa couplings for twisted sectors containing massless states may now be calculated using the approach described in section 2. Four types of contribution to the Yukawa coupling arise for the $\mathbf{Z}_{2} \times \mathbf{Z}_{6}$ orbifold and also for the $\mathbf{Z}_{3} \times \mathbf{Z}_{6}, \mathbf{Z}_{2} \times \mathbf{Z}_{6}^{\prime}$ and $\mathbf{Z}_{6} \times \mathbf{Z}_{6}$ orbifolds. First, if $\mathcal{Z}_{i}$ of (2.12) reduces to a two point function because the $i$-th complex plane is unrotated in one of the three twisted sectors then we may normalize $\mathcal{Z}_{i}$ to 1 . On the other hand, if all the three twist fields in (2.12) are non-trivial then the contribution to $S \lambda i_{c l}$ takes one of three forms. When all three twists are $e \lambda 2 \pi i / 3$, then

$$
S \lambda i_{c l}=\frac{R \lambda 2_{2 i-1}}{8 \pi \sqrt{3}}\left[\left(2 m_{2 i-1}-2 n_{2 i-1}+3 k_{2 i-1}+6 k_{2 i}\right) \lambda 2+3 k_{2 i-1} \lambda 2\right]
$$

where $i=1,2$ or 3 , the arbitrary integers $k_{2 i-1}$ and $k_{2 i}$ derive from the freedom in the choice of the space group elements associated with particular fixed tori, and the integers $m_{2 i-1}$ and $n_{2 i-1}$ characterize any two of the fixed tori involved. The leading contribution to the Yukawa coupling in (2.13) is obtained by minimizing $S \lambda i_{c l}$ by varying the arbitrary integers $k_{2 i-1}$ and $k_{2 i}$. In the case of (3.14), the 
minimum value of $S \lambda i_{c l}$ is given by

$$
\begin{aligned}
\left(S_{c l} \lambda i\right)_{\text {min }} & =0, \quad m_{2 i-1}-n_{2 i-1}=0 \\
& =\frac{2 R_{2 i-1} \lambda 2}{4 \pi \sqrt{3}}, \quad m_{2 i-1}-n_{2 i-1}= \pm 1, \pm 2 .
\end{aligned}
$$

The corresponding moduli dependent suppression factor in the Yukawa coupling is $\exp \left(-S \lambda i_{c l}\right)_{\min }$

For one twist of $e \lambda 4 \pi i / 3$ and two twists of $e \lambda 2 \pi i / 6$,

$$
S \lambda i_{c l}=\frac{R \lambda 2_{2 i-1}}{16 \pi \sqrt{3}}\left[\left(2 n_{2 i-1}+3 k_{2 i-1}+6 k_{2 i}\right) \lambda 2+3 k_{2 i-1} \lambda 2\right]
$$

where $n_{2 i-1}$ characterizes the fixed torus in the sector where the twist on $X_{i}$ is $e \lambda 4 \pi i / 3$. In this case, the minimum value of $S \lambda i_{c l}$ is given by

$$
\begin{aligned}
\left(S_{c l} \lambda i\right)_{\min } & =0, \quad n_{2 i-1}=0 \\
& =\frac{R_{2 i-1} \lambda 2}{4 \pi \sqrt{3}}, \quad n_{2 i-1}= \pm 1 .
\end{aligned}
$$

Finally, for twists of $e \lambda 2 \pi i / 6, e \lambda 2 \pi i / 3$ and -1 ,

$S \lambda i_{c l}=\frac{R \lambda 2_{2 i-1}}{16 \pi \sqrt{3}}\left[\left(2 n_{2 i-1}+3 p_{2 i}+6 k_{2 i}\right) \lambda 2+3\left(2 n_{2 i-1}+3 p_{2 i}-2 p_{2 i-1}+6 k_{2 i}+4 k_{2 i-1}\right) \lambda 2\right]$

where $n_{2 i-1}$ characterizes the fixed torus in the sector where the twist on $X_{i}$ is $e \lambda 2 \pi i / 3$, and $p_{2 i-1}$ and $p_{2 i}$ characterize the fixed torus in the sector where the twist is -1 . In this case, the minimum value of $S \lambda i_{c l}$ is given by

$$
\begin{aligned}
\left(S_{c l} \lambda i\right)_{\min } & =0, \quad n_{2 i-1}=p_{2 i-1}=p_{2 i}=0 \\
& =\frac{3 R_{2 i-1} \lambda 2}{4 \pi \sqrt{3}}, \quad n_{2 i-1}=0, p_{2 i-1}, p_{2 i} \text { not both zero } \\
& =\frac{4 R_{2 i-1} \lambda 2}{4 \pi \sqrt{3}}, \quad n_{2 i-1}= \pm 1, p_{2 i-1}=p_{2 i}=0 \\
& =\frac{R_{2 i-1} \lambda 2}{4 \pi \sqrt{3}},
\end{aligned}
$$

The suppression factors for the non-zero Yukawa couplings amongst the twisted 
sectors of the $\mathbf{Z}_{2} \times \mathbf{Z}_{6}$ orbifold are tabulated in table 4 . Corresponding results for the other $\mathbf{Z}_{M} \times \mathbf{Z}_{N}$ orbifolds are given in the appendix. For the $\mathbf{Z}_{2} \times \mathbf{Z}_{4}$ and $\mathbf{Z}_{4} \times \mathbf{Z}_{4}$ orbifolds, the only non-trivial suppression factor arises when the twists on $X_{i}$ are $e \lambda 2 \pi i / 4, e \lambda 2 \pi i / 4$ and -1 . Then,

$$
S \lambda i_{c l}=\frac{R \lambda 2_{2 i-1}}{16 \pi}\left[\left(2 n_{2 i}+2 p_{2 i-1}-p_{2 i}+4 k_{2 i-1}+2 k_{2 i}\right) \lambda 2+\left(p_{2 i}-2 k_{2 i}\right) \lambda 2\right]
$$

where $n_{2 i}$ characterizes the fixed torus in one of the sectors where the twist on $X_{i}$ is $e \lambda 2 \pi i / 4$ and $p_{2 i-1}$ and $p_{2 i}$ characterize the fixed torus in the sector where the twist is -1 . In this case, the minimum value of $S \lambda i_{c l}$ is given by

$$
\begin{aligned}
\left(S_{c l} \lambda i\right)_{\min } & =\frac{R_{2 i-1} \lambda 2}{8 \pi}, \quad p_{2 i}=1 \\
& =0, \quad p_{2 i}=0, n_{2 i}+p_{2 i-1}=0(\bmod 2) \\
& =\frac{R_{2 i-1} \lambda 2}{4 \pi}, \quad p_{2 i}=0, n_{2 i}+p_{2 i-1}=1(\bmod 2) .
\end{aligned}
$$

For all cases other than the $\mathbf{Z}_{2} \times \mathbf{Z}_{4}$ and $\mathbf{Z}_{4} \times \mathbf{Z}_{4}$ orbifolds, the suppression factors are written in the form $e \lambda-\left(\lambda_{2 i-1} R_{2 i-1} \lambda 2 / 4 \pi \sqrt{3}\right)$, and for the $\mathbf{Z}_{2} \times \mathbf{Z}_{4}$ and $\mathbf{Z}_{4} \times \mathbf{Z}_{4}$ orbifolds in the form $e \lambda-\left(\lambda_{2 i-1} R_{2 i-1} \lambda 2 / 16 \pi\right)$. The suppression factors arising from (3.19) are summarized by

$$
\lambda_{i}=\mu\left(n_{2 i-1}, p_{2 i-1}, p_{2 i}\right),
$$

where

$$
\begin{aligned}
& \mu\left(n_{2 i-1}, p_{2 i-1} p_{2 i}\right)=0, \quad n_{2 i-1}=p_{2 i-1}=p_{2 i}=0 \\
& =3, \quad n_{2 i-1}=0, p_{2 i-1}, p_{2 i} \text { not both zero } \\
& =4, \quad n_{2 i-1}= \pm 1, p_{2 i-1}=p_{2 i}=0 \\
& =1, \quad n_{2 i-1}= \pm 1, p_{2 i-1}, p_{2 i} \text { not both zero, }
\end{aligned}
$$

where $n_{2 i-1}$ characterizes the fixed torus in the sector where the twist on $X_{i}$ is $e \lambda 2 \pi i / 3$, and $p_{2 i-1}$ and $p_{2 i}$ characterize the fixed torus in the sector where the 
twist is -1 . The suppression factors arising from (3.21) are summarized by

$$
\lambda_{i}=\rho\left(n_{2 i}, p_{2 i-1}, p_{2 i}\right),
$$

where

$$
\begin{aligned}
\rho\left(n_{2 i}, p_{2 i-1}, p_{2 i}\right) & =2, & & p_{2 i}=1 \\
& =0, & & p_{2 i}=0, n_{2 i}+p_{2 i-1}=0(\bmod 2) \\
& =4, & & p_{2 i}=0, n_{2 i}+p_{2 i-1}=1(\bmod 2) .
\end{aligned}
$$

The overall moduli and fixed tori independent normalization is easily obtained from the formula derived for $\mathbf{Z}_{N}$ orbifolds in terms of twists in the two-dimensional subspaces [11] which also applies here.

\section{Conclusions}

The moduli dependence of the Yukawa couplings amongst twisted sectors has been investigated for all $\mathbf{Z}_{M} \times \mathbf{Z}_{N}$ orbifolds with the point group realized in the simplest way by Coxeter elements acting on a direct sum of two-dimensional root lattices. All Yukawa couplings have a moduli dependence of the general form (3.14), (3.16), (3.18) or (3.20). A tabulation has been made of the moduli dependent suppression factors for all the non-zero Yukawa couplings. For any definite model, with specified point group embeddings and Wilson lines, the results of this paper may be employed to study suppression factors in the quark and lepton mass matrices.

\section{ACKNOWLEDGEMENTS}

This work was supported in part by S.E.R.C. We would like to thank S. Stieberger for a helpful communication. 


\section{REFERENCES}

1. L. Dixon, J. A. Harvey, C. Vafa and E. Witten, Nucl. Phys. B261 (1985) 678; B274 (1986) 285.

2. A. Font, L. E. Ibanez, F. Quevedo and A. Sierra, Nucl. Phys. B331 (1991) 421.

3. L. Dixon, D. Friedan, E. Martinec and S. Shenker, Nucl. Phys. B282 (1987) 13.

4. S. Hamidi, and C. Vafa, Nucl. Phys. B279 (1987) 465.

5. L. E. Ibanez, Phys. Lett. B181 (1986) 269.

6. J. A. Casas and C. Munoz, Nucl. Phys. B332 (1990) 189

7. J. A. Casas, F. Gomez and C. Munoz, Phys. Lett. B251(1990) 99

8. J. A. Casas, F. Gomez and C. Munoz, CERN preprint, TH6194/91.

9. T. Kobayashi and N. Ohtsubu, Kanazawa preprint, DPKU-9103.

10. T. T. Burwick, R. K. Kaiser and H. F. Muller, Nucl. Phys. B355 (1991) 689

11. J. Erler, D. Jungnickel and J. Lauer, Phys. Rev D45 (1992) 3651; S. Stieberger, D. Jungnickel, J. Lauer and M. Spalinski, preprint, MPI-Ph/9224; J. Erler, D. Jungnickel, M. Spalinski and S. Stieberger, preprint, MPI$\mathrm{Ph} / 92-56$.

12. D. Bailin, A. Love and W. A. Sabra, Mod. Phys. Lett A6 (1992) 3607.

13. S. Stieberger, preprint, TUM-TH-151/92.

14. V. S. Kaplunovsky, Nucl. Phys. B307 (1988) 145; L. J. Dixon, V. S. Kaplunovsky and J. Louis, Nucl. Phys. B355 (1991) 649; J. P. Derenddinger, S. Ferrara, C. Kounas and F. Zwirner, Nucl. Phys. B372 (1992) 145, Phys. Lett. B271 (1991) 307. 
15. I. Antoniadis, J. Ellis, R. Lacaze and D. V. Nanopoulos, CERN-TH 6136/91; S. Kalara, J. L. Lopez and D. V. Nanopoulos, Phys. Lett. B269 (1991) 84 .

16. L. E. Ibanez and D. Lüst and G. G. Ross, Phys. Lett. B272 (1991) 251; D. Bailin and A. Love, Phys. Lett. B278 (1992) 125.

17. L. E. Ibanez and D. Lüst, CERN-TH 6380/92.

18. D. Bailin and A. Love, Phys. Lett. B292 (1992) 315.

19. Y. Katsuki, Y. Kawanura, T. Kobayashi and N. Ohtsubo,Y. Ono and K. Tanioka, Nucl. Phys. B341 (1990) 611; D. Narkushevich, N. Olshanetsky and A. Perelonov, Comm. Math. Phys. 111 (1987) 247.

20. T. Kobayashi and N. Ohtsubo, Phys. Lett. B262 (1991) 425.

21. D. J. Gross, J. A. Harvey, E. Martinec and R. Rohm, Nucl. Phys. B267 (1986) 75.

22. A. Font, L. E. Ibanez and F. Quevedo, Phys. Lett. B217 (1989) 272.

23. T. Kobayashi and N. Ohtsubo, Phys. Lett. B245 (1990) 441. 


\section{Table Captions}

Table 1: Point group elements and lattices for $\mathbf{Z}_{M} \times \mathbf{Z}_{N}$ orbifolds. The point group elements $\theta$ and $\omega$ which are of the form $\left(e \lambda 2 \pi i \eta_{1}, e \lambda 2 \pi i \eta_{2}, e \lambda 2 \pi i \eta_{3}\right)$, are specified in the table by $\left(\eta_{1}, \eta_{2}, \eta_{3}\right)$.

Table 2: Fixed points (and tori) for the $\mathbf{Z}_{2} \times \mathbf{Z}_{6}$ orbifold and associated space group elements. The inequivalent fixed points (or tori) are obtained by giving integers $n$ with half integral coefficients the values 0,1 and integers $n$ with third integral coefficients the values $0, \pm 1$. The coefficients $a_{1}, \ldots, a_{6}$ are arbitrary, and for the space group elements $(\alpha, l)$ the lattice vector $(I-\alpha) \Lambda$, where $\Lambda$ is an arbitrary lattice vector, may be added to $l$.

Table 3: Action of $\theta$ and $\omega$ on the fixed tori for the $\mathbf{Z}_{2} \times \mathbf{Z}_{6}$ orbifold.

Table 4: Suppression factors $e \lambda-\lambda_{1} R_{1} \lambda 2 / 4 \pi \sqrt{3} e \lambda-\lambda_{3} R_{3} \lambda 2 / 4 \pi \sqrt{3} e \lambda-\lambda_{5} R_{5} \lambda 2 / 4 \pi \sqrt{3}$ for the $\mathbf{Z}_{2} \times \mathbf{Z}_{6}$ orbifold Yukawa couplings. The values of the integers characterizing fixed points (or tori) for which non-trivial suppression factors occur are indicated. The $\theta \lambda \alpha \omega \lambda \beta$ twisted sector is denoted by $T_{\alpha \beta}$. 
TABLE 1

\begin{tabular}{|c|c|c|c|}
\hline Point Group & $\theta$ & $\omega$ & Lattice \\
\hline $\mathbf{Z}_{2} \times \mathbf{Z}_{2}$ & $(1,0,-1) / 2$ & $(0,1,-1) / 2$ & $S O(4) \lambda 3$ \\
\hline $\mathbf{Z}_{3} \times \mathbf{Z}_{3}$ & $(1,0,-1) / 3$ & $(0,1,-1) / 3$ & $S U(3) \lambda 3$ \\
\hline $\mathbf{Z}_{2} \times \mathbf{Z}_{4}$ & $(1,0,-1) / 2$ & $(0,1,-1) / 4$ & $S O(4) \times S O(5) \lambda 2$ \\
\hline $\mathbf{Z}_{4} \times \mathbf{Z}_{4}$ & $(1,0,-1) / 4$ & $(0,1,-1) / 4$ & $S O(5) \lambda 3$ \\
\hline $\mathbf{Z}_{2} \times \mathbf{Z}_{6}$ & $(1,0,-1) / 2$ & $(0,1,-1) / 6$ & $S O(4) \times G_{2} \lambda 2$ \\
\hline $\mathbf{Z}_{2} \times \mathbf{Z}_{6}^{\prime}$ & $(1,0,-1) / 2$ & $(1,1,-2) / 6$ & $G_{2} \lambda 3$ \\
\hline $\mathbf{Z}_{3} \times \mathbf{Z}_{6}$ & $(1,0,-1) / 3$ & $(0,1,-1) / 6$ & $S U(3) \times G_{2} \lambda 2$ \\
\hline $\mathbf{Z}_{6} \times \mathbf{Z}_{6}$ & $(1,0,-1) / 6$ & $(0,1,-1) / 6$ & $G_{2} \lambda 3$ \\
\hline
\end{tabular}

TABLE 2

\begin{tabular}{|c|c|c|}
\hline twisted sector & Fixed point or torus & $l$ \\
\hline$\theta$ & $\frac{n_{1}}{2} e_{1}+\frac{n_{2}}{2} e_{2}+\frac{n_{5}}{2} e_{5}+\frac{n_{6}}{2} e_{6}+a_{3} e_{3}+a_{4} e_{4}$ & $n_{1} e_{1}+n_{2} e_{2}+n_{5} e_{5}+n_{6} e_{6}$ \\
\hline$\omega$ & $a_{1} e_{1}+a_{2} e_{2}$ & 0 \\
\hline$\omega \lambda 2$ & $-\frac{n_{3}}{3} e_{4}+\frac{n_{5}}{3} e_{6}+a_{1} e_{1}+a_{2} e_{2}$ & $n_{3} e_{3}+n_{5} e_{5}$ \\
\hline$\omega \lambda 3$ & $\frac{n_{3}}{2} e_{3}+\frac{n_{4}}{2} e_{4}+\frac{n_{5}}{2} e_{5}+\frac{n_{6}}{2} e_{6}+a_{1} e_{1}+a_{2} e_{2}$ & $n_{3} e_{3}+n_{4} e_{4}+n_{5} e_{5}+n_{6} e_{6}$ \\
\hline$\omega \lambda 4$ & $-\frac{n_{3}}{3} e_{4}+\frac{n_{5}}{3} e_{6}+a_{1} e_{1}+a_{2} e_{2}$ & $-n_{3} e_{3}-n_{5} e_{5}$ \\
\hline$\omega \lambda 5$ & $a_{1} e_{1}+a_{2} e_{2}$ & 0 \\
\hline$\theta \omega$ & $-\frac{n_{1}}{2} e_{1}-\frac{n_{2}}{2} e_{2}-\frac{n_{5}}{3} e_{6}$ & $n_{1} e_{1}+n_{2} e_{2}+n_{5} e_{5}$ \\
\hline$\theta \omega \lambda 2$ & $-\frac{n_{1}}{2} e_{1}-\frac{n_{2}}{2} e_{2}-\frac{n_{3}}{3} e_{4}$ & $n_{1} e_{1}+n_{2} e_{2}+n_{3} e_{3}$ \\
\hline$\theta \omega \lambda 3$ & $\frac{n_{1}}{2} e_{1}+\frac{n_{2}}{2} e_{2}+\frac{n_{3}}{2} e_{3}+\frac{n_{4}}{2} e_{4}+a_{5} e_{5}+a_{6} e_{6}$ & $n_{1} e_{1}+n_{2} e_{2}+n_{3} e_{3}+n_{4} e_{4}$ \\
\hline
\end{tabular}


TABLE 3

\begin{tabular}{|c|c|c|}
\hline twisted sector & Action of $\theta$ & Action of $\omega$ \\
\hline$\theta$ & $I$ & $\left(n_{5}, n_{6}\right) \rightarrow\left(n_{6}, n_{5}+n_{6}\right)$ \\
\hline$\omega$ & $I$ & $I$ \\
\hline$\omega \lambda 2$ & $n_{5} \rightarrow-n_{5}$ & $\left(n_{3}, n_{5}\right) \rightarrow\left(-n_{3},-n_{5}\right)$ \\
\hline$\omega \lambda 3$ & $I$ & $\left(n_{3}, n_{4}, n_{5}, n_{6}\right) \rightarrow\left(n_{3}+n_{4}, n_{3}, n_{6}, n_{5}+n_{6}\right)$ \\
\hline$\omega \lambda 4$ & $n_{5} \rightarrow-n_{5}$ & $\left(n_{3}, n_{5}\right) \rightarrow\left(-n_{3},-n_{5}\right)$ \\
\hline$\omega \lambda 5$ & $I$ & $I$ \\
\hline$\theta \omega$ & $n_{5} \rightarrow-n_{5}$ & $n_{3} \rightarrow-n_{3}$ \\
\hline$\theta \omega \lambda 2$ & $I$ & $\left(n_{3}, n_{4}\right) \rightarrow\left(n_{4}, n_{3}+n_{4}\right)$ \\
\hline$\theta \omega \lambda 3$ & $I$ &
\end{tabular}

TABLE 4

\begin{tabular}{|c|c|c|c|}
\hline Yukawa Coupling & $\lambda_{1}$ & $\lambda_{2}$ & $\lambda_{3}$ \\
\hline$T_{01} T_{12} T_{13}$ & 0 & $\mu\left(n_{3}, p_{3}, p_{4}\right)$ & 0 \\
\hline$T_{02} T_{11} T_{13}$ & 0 & $\mu\left(n_{3}, p_{3}, p_{4}\right)$ & 0 \\
\hline$T_{03} T_{10} T_{13}$ & 0 & 0 & 0 \\
\hline$T_{04} T_{10} T_{12}$ & 0 & 0 & $\mu\left(n_{5}, p_{5}, p_{6}\right)$ \\
\hline$T_{04} T_{11} T_{11}$ & 0 & 1 for $n_{3}\left(T_{04}\right) \neq 0$ & 2 for $m_{5}\left(T_{11}\right)-n_{5}\left(T_{11}\right) \neq 0$ \\
\hline$T_{05} T_{10} T_{11}$ & 0 & 0 & $\mu\left(n_{5}, p_{5}, p_{6}\right)$ \\
\hline
\end{tabular}




\section{APPENDIX}

$\mathbf{Z}_{2} \times \mathbf{Z}_{2}$ Orbifold

Lattice : $S O(4) \lambda 3$

$$
\theta=(C(S O(4)), I, C(S O(4))), \quad \omega=(I, C(S O(4)), C(S O(4))) .
$$

The fixed tori and associated space group elements are given with the conventions of table 2 by

\begin{tabular}{|c|c|c|}
\hline twisted sector & Fixed point or torus & $l$ \\
\hline$\theta$ & $\frac{n_{1}}{2} e_{1}+\frac{n_{2}}{2} e_{2}+\frac{n_{5}}{2} e_{5}+\frac{n_{6}}{2} e_{6}+a_{3} e_{3}+a_{4} e_{4}$ & $n_{1} e_{1}+n_{2} e_{2}+n_{5} e_{5}+n_{6} e_{6}$ \\
\hline$\omega$ & $\frac{n_{3}}{2} e_{3}+\frac{n_{4}}{2} e_{4}+\frac{n_{5}}{2} e_{5}+\frac{n_{6}}{2} e_{6}+a_{1} e_{1}+a_{2} e_{2}$ & $n_{3} e_{3}+n_{4} e_{4}+n_{5} e_{5}+n_{6} e_{6}$ \\
\hline$\theta \omega$ & $\frac{n_{1}}{2} e_{1}+\frac{n_{2}}{2} e_{2}+\frac{n_{3}}{2} e_{3}+\frac{n_{4}}{2} e_{4}+a_{5} e_{5}+a_{6} e_{6}$ & $n_{1} e_{1}+n_{2} e_{2}+n_{3} e_{3}+n_{4} e_{4}$ \\
\hline
\end{tabular}

The only non-zero Yukawa couplings is $T_{01} T_{10} T_{11}$, where $T_{\alpha \beta}$ denotes the $\theta \lambda \alpha \omega \lambda \beta$ twisted sector, and since no complex plane is twisted by all three twist fields there is no moduli dependence.

$\underline{\mathbf{Z}_{3} \times \mathbf{Z}_{3} \text { Orbifold }}$

Lattice $S U(3) \lambda 3$

Already discussed in [12].

$\underline{\mathbf{Z}_{2} \times \mathbf{Z}_{4} \text { Orbifold }}$

lattice $S O(4) \times S O(5) \times S O(5)$

In the orthonormal basis,

$$
\begin{array}{ll}
\theta X_{1}=-X_{1}, & \omega X_{1}=X_{1}, \\
\theta X_{2}=X_{2}, & \omega X_{2}=e \lambda 2 \pi i / 4 X_{2}, \\
\theta X_{3}=-X_{3}, & \omega X_{3}=e \lambda-2 \pi i / 4 X_{3} .
\end{array}
$$


In the lattice basis,

$$
\theta=(C(S O(4)), I, C \lambda 2(S O(5))), \quad \omega=(I, C(S O(5)), C \lambda-1(S O(5)))
$$

so that

$$
\begin{array}{lll}
\theta e_{1}=-e_{1}, & \theta e_{3}=e_{3}, & \theta e_{5}=-e_{5}, \\
\theta e_{2}=-e_{2}, & \theta e_{4}=e_{4}, & \theta e_{6}=-e_{6}
\end{array}
$$

and

$$
\begin{aligned}
& \omega e_{1}=e_{1}, \quad \omega e_{3}=e_{3}+2 e_{4}, \quad \omega e_{5}=-e_{5}-2 e_{6}, \\
& \omega e_{2}=e_{2}, \quad \omega e_{4}=-e_{3}-e_{4}, \quad \omega e_{6}=e_{5}+e_{6} .
\end{aligned}
$$

The independent deformation parameters (moduli) are $R_{1}, R_{2}, \cos \theta_{12}, R_{3}$ and $R_{5}$, where

$$
\left|e_{1}\right| \equiv R_{1}, \quad\left|e_{2}\right| \equiv R_{2}, \quad\left|e_{3}\right|=\sqrt{2}\left|e_{4}\right| \equiv R_{3}, \quad\left|e_{5}\right|=\sqrt{2}\left|e_{6}\right| \equiv R_{5},
$$

also,

$$
\cos \theta_{34}=\cos \theta_{56}=-\frac{1}{\sqrt{2}}
$$

with all other $\cos \theta_{i j}$ except $\cos \theta_{12}$ zero. A suitable choice of orthonormal basis $\tilde{e}_{1}, \ldots, \tilde{e}_{6}$ is given by

$$
\begin{aligned}
& e_{1}=R_{1} \tilde{e}_{1} \\
& e_{3}=R_{3} \tilde{e}_{3}, \quad e_{5}=R_{5} \tilde{e}_{5} \\
& e_{2}=R_{2}\left(\cos \theta_{12} \tilde{e}_{1}+\sin \theta_{12} \tilde{e}_{2}\right), \quad e_{4}=\frac{R_{3}}{2}\left(-\tilde{e}_{3}+\tilde{e}_{4}\right), \quad e_{6}=\frac{R_{5}}{2}\left(-\tilde{e}_{5}+\tilde{e}_{6}\right) .
\end{aligned}
$$

The fixed points or tori and associated space group elements are given with the conventions of table 2 by 


\begin{tabular}{|c|c|c|}
\hline twisted sector & Fixed point or torus & $l$ \\
\hline$\theta$ & $\frac{n_{1}}{2} e_{1}+\frac{n_{2}}{2} e_{2}+\frac{n_{5}}{2} e_{5}+\frac{n_{6}}{2} e_{6}+a_{3} e_{3}+a_{4} e_{4}$ & $n_{1} e_{1}+n_{2} e_{2}+n_{5} e_{5}+n_{6} e_{6}$ \\
\hline$\omega$ & $a_{1} e_{1}+a_{2} e_{2}-\frac{n_{4}}{2} e_{3}+\frac{n_{6}}{2} e_{5}$ & $n_{4} e_{4}-n_{6} e_{6}$ \\
\hline$\omega \lambda 2$ & $a_{1} e_{1}+a_{2} e_{2}+\frac{n_{3}}{2} e_{3}+\frac{n_{4}}{2} e_{4}+\frac{n_{5}}{2} e_{5}+\frac{n_{6}}{2} e_{6}$ & $n_{3} e_{3}+n_{4} e_{4}+n_{5} e_{5}+n_{6} e_{6}$ \\
\hline$\omega \lambda 3$ & $a_{1} e_{1}+a_{2} e_{2}-\frac{n_{4}}{2} e_{3}+\frac{n_{6}}{2} e_{5}$ & $-n_{4} e_{4}+n_{6} e_{6}$ \\
\hline$\theta \omega$ & $\frac{n_{1}}{2} e_{1}+\frac{n_{2}}{2} e_{2}-\frac{n_{4}}{2} e_{3}+\frac{n_{6}}{2} e_{5}$ & $n_{1} e_{1}+n_{2} e_{2}+n_{4} e_{4}+n_{6} e_{6}$ \\
\hline$\theta \omega \lambda 2$ & $\frac{n_{1}}{2} e_{1}+\frac{n_{2}}{2} e_{2}+\frac{n_{3}}{2} e_{3}+\frac{n_{4}}{2} e_{4}+a_{5} e_{5}+a_{6} e_{6}$ & $n_{1} e_{1}+n_{2} e_{2}+n_{3} e_{3}+n_{4} e_{4}$ \\
\hline$\theta \omega \lambda 3$ & $\frac{n_{1}}{2} e_{1}+\frac{n_{2}}{2} e_{2}-\frac{n_{4}}{2} e_{3}+\frac{n_{6}}{2} e_{5}$ & $n_{1} e_{1}+n_{2} e_{2}+n_{6} e_{6}-n_{4} e_{4}$ \\
\hline
\end{tabular}

The action of $\theta$ and $\omega$ on the fixed points (or tori) is given by

\begin{tabular}{|c|c|c|}
\hline Twisted sector & Action of $\theta$ & Action of $\omega$ \\
\hline$\theta$ & $I$ & $n_{5} \rightarrow n_{5}+n_{6}$ \\
\hline$\omega, \omega \lambda 3$ & $I$ & $I$ \\
\hline$\omega \lambda 2$ & $I$ & $\left(n_{3}, n_{5}\right) \rightarrow\left(n_{3}+n_{4}, n_{5}+n_{6}\right)$ \\
\hline$\theta \omega, \theta \omega \lambda 3$ & $I$ & $I$ \\
\hline$\theta \omega \lambda 2$ & $I$ & $n_{3} \rightarrow n_{3}+n_{4}$ \\
\hline
\end{tabular}

The moduli dependent suppression factors for the non-zero Yukawa couplings amongst twisted sectors containing massless states written in the form $e \lambda-\lambda_{2 i-1} R_{2 i-1} \lambda 2 / 16 \pi$ are given by

\begin{tabular}{|c|c|c|c|}
\hline Yukawa Coupling & $\lambda_{1}$ & $\lambda_{2}$ & $\lambda_{3}$ \\
\hline$T_{01} T_{11} T_{12}$ & 0 & $\rho\left(n_{4}, p_{3}, p_{4}\right)$ & 0 \\
\hline$T_{02} T_{10} T_{12}$ & 0 & 0 & 0 \\
\hline$T_{03} T_{10} T_{11}$ & 0 & 0 & $\rho\left(n_{6}, p_{5}, p_{6}\right)$ \\
\hline$T_{11} T_{11} T_{02}$ & 0 & $\rho\left(n_{4}, p_{3}, p_{4}\right)$ & $\rho\left(n_{6}, p_{5}, p_{6}\right)$ \\
\hline
\end{tabular}


$\underline{\mathbf{Z}_{4} \times \mathbf{Z}_{4} \text { Orbifold }}$

Lattice $S O(5) \times S O(5) \times S O(5)$

In the orthonormal basis,

$$
\begin{array}{ll}
\theta X_{1}=e \lambda 2 \pi i / 4 X_{1}, & \omega X_{1}=X_{1}, \\
\theta X_{2}=X_{2}, & \omega X_{2}=e \lambda 2 \pi i / 4 X_{2}, \\
\theta X_{3}=e \lambda-2 \pi i / 4 X_{3}, & \omega X_{3}=e \lambda-2 \pi i / 4 X_{3} .
\end{array}
$$

In the lattice basis,

$$
\theta=(C(S O(5)), I, C \lambda-1(S O(5))), \quad \omega=(I, C(S O(5)), C \lambda-1(S O(5)))
$$

so that

$$
\begin{aligned}
& \theta e_{1}=e_{1}+2 e_{2}, \quad \theta e_{3}=e_{3}, \quad \theta e_{5}=-e_{5}-2 e_{6}, \\
& \theta e_{2}=-e_{1}-e_{2}, \quad \theta e_{4}=e_{4}, \quad \theta e_{6}=e_{5}+e_{6}
\end{aligned}
$$

and

$$
\begin{array}{lll}
\omega e_{1}=e_{1}, & \omega e_{3}=e_{3}+2 e_{4}, & \omega e_{5}=-e_{5}-2 e_{6}, \\
\omega e_{2}=e_{2}, & \omega e_{4}=-e_{3}-e_{4}, & \omega e_{6}=e_{5}+e_{6} .
\end{array}
$$

The independent deformation parameters (moduli) are $R_{1}, R_{3}$ and $R_{5}$, where

$$
\left|e_{1}\right|=\sqrt{2}\left|e_{2}\right| \equiv R_{1}, \quad\left|e_{3}\right|=\sqrt{2}\left|e_{4}\right| \equiv R_{3}, \quad\left|e_{5}\right|=\sqrt{2}\left|e_{6}\right| \equiv R_{5},
$$

also

$$
\cos \theta_{12}=\cos \theta_{34}=\cos \theta_{56}=-\frac{1}{\sqrt{2}}
$$

with all other $\cos \theta_{i j}$ zero. A suitable choice of orthonormal basis $\tilde{e}_{1}, \ldots, \tilde{e}_{6}$ is given by

$$
\begin{array}{lll}
e_{1}=R_{1} \tilde{e}_{1}, & e_{3}=R_{3} \tilde{e}_{3}, & e_{5}=R_{5} \tilde{e}_{5}, \\
e_{2}=-\frac{R_{1}}{2}\left(\tilde{e}_{1}-\tilde{e}_{2}\right), & e_{4}=-\frac{R_{3}}{2}\left(\tilde{e}_{3}-\tilde{e}_{4}\right), & e_{6}=-\frac{R_{5}}{2}\left(\tilde{e}_{5}-\tilde{e}_{6}\right) .
\end{array}
$$


The fixed points or tori and associated space group elements are given with the conventions of table 2 by

\begin{tabular}{|c|c|c|}
\hline twisted sector & Fixed point or torus & $l$ \\
\hline$\theta$ & $-\frac{n_{2}}{2} e_{1}+\frac{n_{6}}{2} e_{5}+a_{3} e_{3}+a_{4} e_{4}$ & $n_{2} e_{2}+n_{6} e_{6}$ \\
\hline$\omega$ & $a_{1} e_{1}+a_{2} e_{2}-\frac{n_{4}}{2} e_{3}+\frac{n_{6}}{2} e_{5}$ & $n_{4} e_{4}+n_{6} e_{6}$ \\
\hline$\omega \lambda 2$ & $a_{1} e_{1}+a_{2} e_{2}+\frac{n_{3}}{2} e_{3}+\frac{n_{4}}{2} e_{4}+\frac{n_{5}}{2} e_{5}+\frac{n_{6}}{2} e_{6}$ & $n_{3} e_{3}+n_{4} e_{4}+n_{5} e_{5}+n_{6} e_{6}$ \\
\hline$\omega \lambda 3$ & $a_{1} e_{1}+a_{2} e_{2}+\frac{n_{4}}{2} e_{3}-\frac{n_{6}}{2} e_{5}$ & $n_{4} e_{4}+n_{6} e_{6}$ \\
\hline$\theta \omega$ & $-\frac{n_{2}}{2} e_{1}-\frac{n_{4}}{2} e_{3}+\frac{n_{5}}{2} e_{5}+\frac{n_{6}}{2} e_{6}$ & $n_{2} e_{2}+n_{4} e_{4}+n_{5} e_{5}+n_{6} e_{6}$ \\
\hline$\theta \omega \lambda 2$ & $-\frac{n_{2}}{2} e_{1}+\frac{n_{3}}{2} e_{3}+\frac{n_{4}}{2} e_{4}-\frac{n_{6}}{2} e_{5}$ & $n_{2} e_{2}+n_{4} e_{4}+n_{3} e_{3}+n_{6} e_{6}$ \\
\hline$\theta \omega \lambda 3$ & $-\frac{n_{2}}{2} e_{1}+\frac{n_{4}}{2} e_{3}+a_{5} e_{5}+a_{6} e_{6}$ & $n_{2} e_{2}+n_{4} e_{4}$ \\
\hline$\theta \lambda 2$ & $\frac{n_{1}}{2} e_{1}+\frac{n_{2}}{2} e_{2}+a_{3} e_{3}+a_{4} e_{4}+\frac{n_{5}}{2} e_{5}+\frac{n_{6}}{2} e_{6}$ & $n_{1} e_{1}+n_{2} e_{2}+n_{5} e_{5}+n_{6} e_{6}$ \\
\hline$\theta \lambda 3$ & $\frac{n_{2}}{2} e_{1}+a_{3} e_{3}+a_{4} e_{4}-\frac{n_{6}}{2} e_{5}$ & $n_{2} e_{2}+n_{6} e_{6}$ \\
\hline$\theta \lambda 2 \omega$ & $\frac{n_{1}}{2} e_{1}+\frac{n_{2}}{2} e_{2}-\frac{n_{4}}{2} e_{3}-\frac{n_{6}}{2} e_{5}$ & $n_{1} e_{1}+n_{2} e_{2}+n_{4} e_{4}+n_{6} e_{6}$ \\
\hline$\theta \lambda 2 \omega \lambda 2$ & $\frac{n_{1}}{2} e_{1}+\frac{n_{2}}{2} e_{2}+\frac{n_{3}}{2} e_{3}+\frac{n_{4}}{2} e_{4}+a_{5} e_{5}+a_{6} e_{6}$ & $n_{1} e_{1}+n_{2} e_{2}+n_{3} e_{3}+n_{4} e_{4}$ \\
\hline$\theta \lambda 3 \omega$ & $\frac{n_{2}}{2} e_{1}-\frac{n_{4}}{2} e_{3}+a_{5} e_{5}+a_{6} e_{6}$ & $n_{2} e_{2}+n_{4} e_{4}$ \\
\hline$\theta \lambda 2 \omega \lambda 3$ & $\frac{n_{1}}{2} e_{1}+\frac{n_{2}}{2} e_{2}+\frac{n_{4}}{2} e_{3}+\frac{n_{6}}{2} e_{5}$ & $n_{1} e_{1}+n_{2} e_{2}+n_{4} e_{4}+n_{6} e_{6}$ \\
\hline$\theta \lambda 3 \omega \lambda 2$ & $\frac{n_{2}}{2} e_{1}+\frac{n_{3}}{2} e_{3}+\frac{n_{4}}{2} e_{4}+\frac{n_{6}}{2} e_{5}$ & $n_{2} e_{2}+n_{3} e_{3}+n_{4} e_{4}+n_{6} e_{6}$ \\
\hline$\theta \lambda 3 \omega \lambda 3$ & $\frac{n_{2}}{2} e_{1}+\frac{n_{4}}{2} e_{3}+\frac{n_{5}}{2} e_{5}+\frac{n_{6}}{2} e_{6}$ & $n_{2} e_{2}+n_{4} e_{4}+n_{5} e_{5}+n_{6} e_{6}$ \\
\hline
\end{tabular}


The action of $\theta$ and $\omega$ on the fixed points (or tori) is given by

\begin{tabular}{|c|c|c|}
\hline twisted sector & Action of theta & Action of omega \\
\hline$\theta, \theta \lambda 3$ & $I$ & $I$ \\
\hline$\omega, \omega \lambda 3$ & $I$ & $I$ \\
\hline$\theta \lambda 3 \omega, \theta \omega \lambda 3$ & $I$ & $n_{5} \rightarrow n_{5}+n_{6}$ \\
\hline$\theta \omega, \theta \lambda 3 \omega \lambda 3$ & $n_{5} \rightarrow n_{5}+n_{6}$ & $n_{3} \rightarrow n_{3}+n_{4}$ \\
\hline$\theta \omega \lambda 2, \theta \lambda 3 \omega \lambda 2$ & $I$ & $I$ \\
\hline$\theta \lambda 2 \omega, \theta \lambda 2 \omega \lambda 3$ & $n_{1} \rightarrow n_{1}+n_{2}$ & $\left(n_{3}, n_{5}\right) \rightarrow\left(n_{3}+n_{4}, n_{5}+n_{6}\right)$ \\
\hline$\omega \lambda 2$ & $n_{5} \rightarrow n_{5}+n_{6}$ & $n_{5} \rightarrow n_{5}+n_{6}$ \\
\hline$\theta \lambda 2$ & $\left(n_{1}, n_{5}\right) \rightarrow\left(n_{1}+n_{2}, n_{5}+n_{6}\right)$ & $n_{3} \rightarrow n_{3}+n_{4}$ \\
\hline$\theta \lambda 2 \omega \lambda 2$ & $n_{1} \rightarrow n_{1}+n_{2}$ & \\
\hline
\end{tabular}


The moduli dependent suppression factors for the non-zero Yukawa couplings amongst twisted sectors containing massless states written in the form $e \lambda-\lambda_{2 i-1} R_{2 i-1} \lambda 2 / 16 \pi$ are given by

\begin{tabular}{|c|c|c|c|}
\hline Yukawa coupling & $\lambda_{1}$ & $\lambda_{2}$ & $\lambda_{3}$ \\
\hline$T_{01} T_{12} T_{31}$ & 0 & $\rho\left(n_{4}, p_{3}, p_{4}\right)$ & 0 \\
\hline$T_{01} T_{13} T_{30}$ & 0 & 0 & 0 \\
\hline$T_{01} T_{21} T_{22}$ & 0 & $\rho\left(n_{4}, p_{3}, p_{4}\right)$ & 0 \\
\hline$T_{02} T_{11} T_{31}$ & 0 & $\rho\left(n_{4}, p_{3}, p_{4}\right)$ & 0 \\
\hline$T_{02} T_{12} T_{30}$ & 0 & 0 & $\rho\left(n_{6}, p_{5}, p_{6}\right)$ \\
\hline$T_{02} T_{20} T_{22}$ & 0 & 0 & 0 \\
\hline$T_{02} T_{21} T_{21}$ & 0 & $\rho\left(n_{4}, p_{3}, p_{4}\right)$ & $\rho\left(n_{6}, p_{5}, p_{6}\right)$ \\
\hline$T_{03} T_{10} T_{31}$ & 0 & 0 & 0 \\
\hline$T_{03} T_{11} T_{30}$ & 0 & 0 & $\rho\left(n_{6}, p_{5}, p_{6}\right)$ \\
\hline$T_{03} T_{20} T_{21}$ & 0 & 0 & $\rho\left(n_{6}, p_{5}, p_{6}\right)$ \\
\hline$T_{10} T_{12} T_{22}$ & $\rho\left(n_{2}, p_{1}, p_{2}\right)$ & 0 & 0 \\
\hline$T_{10} T_{13} T_{21}$ & $\rho\left(n_{2}, p_{1}, p_{2}\right)$ & 0 & 0 \\
\hline$T_{11} T_{12} T_{21}$ & $\rho\left(n_{2}, p_{1}, p_{2}\right)$ & $\rho\left(n_{4}, p_{3}, p_{4}\right)$ & $\rho\left(n_{6}, p_{5}, p_{6}\right)$ \\
\hline$T_{11} T_{11} T_{22}$ & $\rho\left(n_{2}, p_{1}, p_{2}\right)$ & $\rho\left(n_{4}, p_{3}, p_{4}\right)$ & 0 \\
\hline$T_{11} T_{13} T_{20}$ & $\rho\left(n_{2}, p_{1}, p_{2}\right)$ & 0 & 0 \\
\hline$T_{12} T_{12} T_{20}$ & $\rho\left(n_{2}, p_{1}, p_{2}\right)$ & 0 & $\rho\left(n_{6}, p_{5}, p_{6}\right)$ \\
\hline
\end{tabular}


$\underline{\mathbf{Z}_{2} \times \mathbf{Z}_{6} \text { Orbifold }}$

Lattice $S O(4) \times G_{2} \lambda 2$

Discussed in section 3 .

$\mathbf{Z}_{2} \times \mathbf{Z}_{6}^{\prime}$ Orbifold

Lattice $G_{2} \lambda 3$

In the orthonormal basis,

$$
\begin{array}{ll}
\theta X_{1}=-X_{1}, & \omega X_{1}=e \lambda 2 \pi i / 6 X_{1}, \\
\theta X_{2}=X_{2}, & \omega X_{2}=e \lambda 2 \pi i / 6 X_{2}, \\
\theta X_{3}=-X_{3}, & \omega X_{3}=e \lambda-2 \pi i / 3 X_{3} .
\end{array}
$$

In the lattice basis,

$$
\theta=\left(C \lambda 3\left(G_{2}\right), I, C \lambda 3\left(G_{2}\right)\right), \quad \omega=\left(C\left(G_{2}\right), C\left(G_{2}\right), C \lambda-2\left(G_{2}\right)\right)
$$

so that

$$
\begin{array}{lll}
\theta e_{1}=-e_{1}, & \theta e_{3}=e_{3}, & \theta e_{5}=-e_{5}, \\
\theta e_{2}=-e_{2}, & \theta e_{4}=e_{4}, & \theta e_{6}=-e_{6} .
\end{array}
$$

and

$$
\begin{array}{ll}
\omega e_{1}=-e_{1}-e_{2}, & \omega e_{3}=-e_{3}-e_{4}, \quad \omega e_{5}=e_{5}+e_{6}, \\
\omega e_{2}=3 e_{1}+2 e_{2}, & \omega e_{4}=3 e_{3}+2 e_{4}, \quad \omega e_{6}=-3 e_{5}-2 e_{6} .
\end{array}
$$

The independent deformation parameters (moduli) are $R_{1}, R_{3}$ and $R_{5}$, where

$$
\left|e_{2}\right|=\sqrt{3}\left|e_{1}\right| \equiv \sqrt{3} R_{1}, \quad\left|e_{4}\right|=\sqrt{3}\left|e_{3}\right| \equiv \sqrt{3} R_{3}, \quad\left|e_{6}\right|=\sqrt{3}\left|e_{5}\right| \equiv \sqrt{3} R_{5}
$$

also,

$$
\cos \theta_{12}=\cos \theta_{34}=\cos \theta_{56}=-\frac{\sqrt{3}}{2}
$$

with all other $\cos \theta_{i j}$ zero. A suitable choice of orthonormal basis $\tilde{e}_{1}, \ldots, \tilde{e}_{6}$ is given 
by
$e_{1}=R_{1} \tilde{e}_{1}$
$e_{3}=R_{3} \tilde{e}_{3}$
$e_{5}=R_{5} \tilde{e}_{5}$
$e_{2}=R_{1}\left(-\frac{3}{2} \tilde{e}_{1}-\frac{\sqrt{3}}{2} \tilde{e}_{2}\right)$
$e_{4}=R_{3}\left(-\frac{3}{2} \tilde{e}_{3}-\frac{\sqrt{3}}{2} \tilde{e}_{4}\right)$
$e_{6}=R_{5}\left(-\frac{3}{2} \tilde{e}_{5}-\frac{\sqrt{3}}{2} \tilde{e}_{6}\right)$

The fixed points or tori and associated space group elements are given with the conventions of table 2 by

\begin{tabular}{|c|c|c|}
\hline Twisted sector & Fixed point or torus & $l$ \\
\hline$\theta$ & $\frac{n_{1}}{2} e_{1}+\frac{n_{2}}{2} e_{2}+\frac{n_{5}}{2} e_{5}+\frac{n_{6}}{2} e_{6}+a_{3} e_{3}+a_{4} e_{4}$ & $n_{1} e_{1}+n_{2} e_{2}+n_{5} e_{5}+n_{6} e_{6}$ \\
\hline$\omega$ & $\frac{n_{5}}{3} e_{6}$ & $n_{5} e_{5}$ \\
\hline$\omega \lambda 2$ & $-\frac{n_{1}}{3} e_{2}-\frac{n_{3}}{3} e_{4}+\frac{n_{5}}{3} e_{6}$ & $n_{1} e_{1}+n_{3} e_{3}-n_{5} e_{5}$ \\
\hline$\omega \lambda 3$ & $\frac{n_{1}}{2} e_{1}+\frac{n_{2}}{2} e_{2}+\frac{n_{3}}{2} e_{3}+\frac{n_{4}}{2} e_{4}+a_{5} e_{5}+a_{6} e_{6}$ & $n_{1} e_{1}+n_{2} e_{2}+n_{3} e_{3}+n_{4} e_{4}$ \\
\hline$\omega \lambda 4$ & $-\frac{n_{1}}{3} e_{2}-\frac{n_{3}}{3} e_{4}+\frac{n_{5}}{3} e_{6}$ & $-n_{1} e_{1}-n_{3} e_{3}+n_{5} e_{5}$ \\
\hline$\omega \lambda 5$ & $\frac{n_{5}}{3} e_{6}$ & $-n_{5} e_{5}$ \\
\hline$\theta \omega$ & $\frac{n_{1}}{3} e_{1}$ & $n_{1} e_{1}$ \\
\hline$\theta \omega \lambda 2$ & $-\frac{n_{3}}{3} e_{4}$ & $n_{3} e_{3}$ \\
\hline$\theta \omega \lambda 3$ & $\frac{n_{3}}{2} e_{3}+\frac{n_{4}}{2} e_{4}+\frac{n_{5}}{2} e_{5}+\frac{n_{6}}{2} e_{6}+a_{1} e_{1}+a_{2} e_{2}$ & $n_{3} e_{3}+n_{4} e_{4}+n_{5} e_{5}+n_{6} e_{6}$ \\
\hline$\theta \omega \lambda 4$ & $-\frac{n_{3}}{3} e_{4}$ & $-n_{3} e_{3}$ \\
\hline$\theta \omega \lambda 5$ & $\frac{n_{1}}{3} e_{2}$ & $-n_{1} e_{1}$ \\
\hline & & \\
\hline
\end{tabular}


The action of $\theta$ and $\omega$ on the fixed points (or tori) is given by

\begin{tabular}{|c|c|c|}
\hline Twisted sector & Action of $\theta$ & Action of $\omega$ \\
\hline$\theta$ & $I$ & $\left(n_{1}, n_{2}, n_{5}, n_{6}\right) \rightarrow\left(n_{1}+n_{2}, n_{1}, n_{5}+n_{6}, n_{5}\right)$ \\
\hline$\omega, \omega \lambda 5$ & $n_{5} \rightarrow-n_{5}$ & $I$ \\
\hline$\omega \lambda 2, \omega \lambda 4$ & $\left(n_{3}, n_{5}\right) \rightarrow\left(-n_{3},-n_{5}\right)$ & $\left(n_{1}, n_{3}\right) \rightarrow\left(-n_{1},-n_{3}\right)$ \\
\hline$\omega \lambda 3$ & $I$ & $\left(n_{1}, n_{2}, n_{3}, n_{4}\right) \rightarrow\left(n_{1}+n_{2}, n_{1}, n_{3}+n_{4}, n_{3}\right)$ \\
\hline$\theta \omega, \theta \omega \lambda 5$ & $n_{1} \rightarrow-n_{1}$ & $n_{1} \rightarrow-n_{1}$ \\
\hline$\theta \omega \lambda 2, \theta \omega \lambda 4$ & $I$ & $n_{3} \rightarrow-n_{3}$ \\
\hline$\theta \omega \lambda 3$ & $I$ & $\left(n_{3}, n_{4}, n_{5}, n_{6}\right) \rightarrow\left(n_{3}+n_{4}, n_{3}, n_{5}+n_{6}, n_{5}\right)$ \\
\hline
\end{tabular}

The moduli dependent suppression factors for the non-zero Yukawa couplings amongst twisted sectors containing massless states are given in the conventions of table 4 by

\begin{tabular}{|c|c|c|c|}
\hline $\begin{array}{c}\text { Yukawa } \\
\text { Coupling }\end{array}$ & $\lambda_{1}$ & $\lambda_{2}$ & $\lambda_{3}$ \\
\hline$T_{01} T_{02} T_{03}$ & $\mu\left(n_{1}, p_{1}, p_{2}\right)$ & $\mu\left(n_{3}, p_{3}, p_{4}\right)$ & 0 \\
\hline$T_{01} T_{11} T_{14}$ & 1 for $n_{1}\left(T_{11}\right) \neq 0$ & 1 for $n_{3}\left(T_{14}\right) \neq 0$ & 1 for $n_{5}\left(T_{01}\right) \neq 0$ \\
\hline$T_{02} T_{02} T_{02}$ & 2 for $m_{1}\left(T_{02}\right)-n_{1}\left(T_{02}\right) \neq 0$ & 2 for $m_{3}\left(T_{02}\right)-n_{3}\left(T_{02}\right) \neq 0$ & 2 for $m_{5}\left(T_{02}\right)-n_{5}\left(T_{02}\right) \neq 0$ \\
\hline$T_{02} T_{10} T_{14}$ & $\mu\left(n_{1}, p_{1}, p_{2}\right)$ & 0 & $\mu\left(n_{5}, p_{5}, p_{6}\right)$ \\
\hline$T_{02} T_{11} T_{13}$ & 0 & $\mu\left(n_{3}, p_{3}, p_{4}\right)$ & $\mu\left(n_{5}, p_{5}, p_{6}\right)$ \\
\hline$T_{03} T_{10} T_{13}$ & 0 & 0 & 0 \\
\hline
\end{tabular}




\section{$\mathbf{Z}_{3} \times \mathbf{Z}_{6}$ Orbifold}

lattice $S U(3) \times G_{2} \lambda 2$

In the orthonormal basis,

$$
\begin{array}{ll}
\theta X_{1}=e \lambda 2 \pi i / 3 X_{1}, & \omega X_{1}=X_{1}, \\
\theta X_{2}=X_{2}, & \omega X_{2}=e \lambda 2 \pi i / 6 X_{2}, \\
\theta X_{3}=e \lambda-2 \pi i / 3 X_{3}, & \omega X_{3}=e \lambda-2 \pi i / 6 X_{3} .
\end{array}
$$

In the lattice basis,

$$
\theta=\left(C(S U(3)), I, C \lambda-2\left(G_{2}\right)\right), \quad \omega=\left(I, C\left(G_{2}\right), C \lambda-1\left(G_{2}\right)\right)
$$

so that

$$
\begin{aligned}
& \theta e_{1}=e_{2}, \quad \theta e_{3}=e_{3}, \quad \theta e_{5}=e_{5}+e_{6}, \\
& \theta e_{2}=-e_{1}-e_{2}, \quad \theta e_{4}=e_{4}, \quad \theta e_{6}=-3 e_{5}-2 e_{6} .
\end{aligned}
$$

and

$$
\begin{aligned}
& \omega e_{1}=e_{1}, \quad \omega e_{3}=-e_{3}-e_{4}, \quad \omega e_{5}=2 e_{5}+e_{6}, \\
& \omega e_{2}=e_{2}, \quad \omega e_{4}=3 e_{3}+2 e_{4}, \quad \omega e_{6}=-3 e_{5}-e_{6} .
\end{aligned}
$$

The independent deformation parameters (moduli) are $R_{1}, R_{3}$ and $R_{5}$, where

$$
\left|e_{2}\right|=\left|e_{1}\right| \equiv R_{1}, \quad\left|e_{4}\right|=\sqrt{3}\left|e_{3}\right| \equiv \sqrt{3} R_{3}, \quad\left|e_{6}\right|=\sqrt{3}\left|e_{5}\right| \equiv \sqrt{3} R_{5}
$$

also,

$$
\cos \theta_{12}=-\frac{1}{2}, \quad \cos \theta_{34}=\cos \theta_{56}=-\frac{\sqrt{3}}{2}
$$

with all other $\cos \theta_{i j}$ zero. A suitable choice of orthonormal basis $\tilde{e}_{1}, \ldots, \tilde{e}_{6}$ is given by

$$
\begin{aligned}
& e_{1}=R_{1} \tilde{e}_{1}, \quad e_{3}=R_{3} \tilde{e}_{3}, \quad e_{5}=R_{5} \tilde{e}_{5}, \\
& e_{2}=R_{1}\left(-\frac{1}{2} \tilde{e}_{1}+\frac{\sqrt{3}}{2} \tilde{e}_{2}\right), \quad e_{4}=R_{3}\left(-\frac{3}{2} \tilde{e}_{3}-\frac{\sqrt{3}}{2} \tilde{e}_{4}\right), \quad e_{6}=R_{5}\left(-\frac{3}{2} \tilde{e}_{5}-\frac{\sqrt{3}}{2} \tilde{e}_{6}\right) .
\end{aligned}
$$

The fixed points or tori and associated group elements are given with the conventions of table 2 by 


\begin{tabular}{|c|c|c|}
\hline Twisted sector & Fixed point or torus & $l$ \\
\hline$\theta$ & $\frac{n_{1}}{3}\left(2 e_{1}+e_{2}\right)+\frac{n_{5}}{3} e_{6}+a_{3} e_{3}+a_{4} e_{4}$ & $n_{1} e_{1}+n_{5} e_{5}$ \\
\hline$\theta \lambda 2$ & $\frac{n_{1}}{3}\left(2 e_{1}+e_{2}\right)+\frac{n_{5}}{3} e_{6}+a_{3} e_{3}+a_{4} e_{4}$ & $-n_{1} e_{1}-n_{5} e_{5}$ \\
\hline$\omega$ & $a_{1} e_{1}+a_{2} e_{2}$ & 0 \\
\hline$\omega \lambda 2$ & $-\frac{n_{3}}{3} e_{4}+\frac{n_{5}}{3} e_{6}+a_{1} e_{1}+a_{2} e_{2}$ & $n_{3} e_{3}+n_{5} e_{5}$ \\
\hline$\omega \lambda 3$ & $\frac{n_{3}}{2} e_{3}+\frac{n_{4}}{2} e_{4}+\frac{n_{5}}{2} e_{5}+\frac{n_{6}}{2} e_{6}+a_{1} e_{1}+a_{2} e_{2}$ & $n_{3} e_{3}+n_{4} e_{4}+n_{5} e_{5}+n_{6} e_{6}$ \\
\hline$\omega \lambda 4$ & $-\frac{n_{3}}{3} e_{4}+\frac{n_{5}}{3} e_{6}+a_{1} e_{1}+a_{2} e_{2}$ & $-n_{3} e_{3}-n_{5} e_{5}$ \\
\hline$\omega \lambda 5$ & $a_{1} e_{1}+a_{2} e_{2}$ & 0 \\
\hline$\theta \omega$ & $\frac{n_{1}}{3}\left(2 e_{1}+e_{2}\right)+\frac{n_{5}}{2} e_{5}+\frac{n_{6}}{2} e_{6}$ & $n_{1} e_{1}+n_{5} e_{5}+n_{6} e_{6}$ \\
\hline$\theta \omega \lambda 2$ & $\frac{n_{1}}{3}\left(2 e_{1}+e_{2}\right)-\frac{n_{3}}{3} e_{4}+\frac{n_{5}}{3} e_{6}$ & $n_{1} e_{1}+n_{3} e_{3}-n_{5} e_{5}$ \\
\hline$\theta \omega \lambda 3$ & $\frac{n_{1}}{3}\left(2 e_{1}+e_{2}\right)+\frac{n_{3}}{2} e_{3}+\frac{n_{4}}{2} e_{4}$ & $n_{1} e_{1}+n_{3} e_{3}+n_{4} e_{4}$ \\
\hline$\theta \omega \lambda 4$ & $\frac{n_{1}}{3}\left(2 e_{1}+e_{2}\right)-\frac{n_{3}}{2} e_{4}+a_{5} e_{5}+a_{6} e_{6}$ & $n_{1} e_{1}-n_{3} e_{3}$ \\
\hline$\theta \omega \lambda 5$ & $\frac{n_{1}}{3}\left(2 e_{1}+e_{2}\right)$ & $n_{1} e_{1}$ \\
\hline$\theta \lambda 2 \omega$ & $\frac{n_{1}}{3}\left(2 e_{1}+e_{2}\right)$ & $-n_{1} e_{1}$ \\
\hline$\theta \lambda 2 \omega \lambda 2$ & $\frac{n_{1}}{3}\left(2 e_{1}+e_{2}\right)-\frac{n_{3}}{3} e_{4}$ & $-n_{1} e_{1}+n_{3} e_{3}$ \\
\hline$\theta \lambda 2 \omega \lambda 3$ & $\frac{n_{1}}{3}\left(2 e_{1}+e_{2}\right)+\frac{n_{3}}{2} e_{3}+\frac{n_{4}}{2} e_{4}$ & $-n_{1} e_{1}+n_{3} e_{3}+n_{4} e_{4}$ \\
\hline$\theta \lambda 2 \omega \lambda 4$ & $\frac{n_{1}}{3}\left(2 e_{1}+e_{2}\right)-\frac{n_{3}}{3} e_{4}+\frac{n_{5}}{3} e_{6}$ & $-n_{1} e_{1}-n_{3} e_{3}+n_{5} e_{5}$ \\
\hline$\theta \lambda 2 \omega \lambda 5$ & $\frac{n_{1}}{3}\left(2 e_{1}+e_{2}\right)+\frac{n_{5}}{2} e_{5}+\frac{n_{6}}{2} e_{6}$ & $-n_{1} e_{1}+n_{5} e_{5}+n_{6} e_{6}$ \\
\hline
\end{tabular}


The action of $\theta$ and $\omega$ on the fixed points (or tori) is given by

\begin{tabular}{|c|c|c|}
\hline Twisted sector & Action of $\theta$ & Action of $\omega$ \\
\hline$\theta, \theta \lambda 2$ & $I$ & $n_{5} \rightarrow-n_{5}$ \\
\hline$\omega, \omega \lambda 5$ & $I$ & $I$ \\
\hline$\omega \lambda 2, \omega \lambda 4$ & $I$ & $\left(n_{3}, n_{5}\right) \rightarrow\left(-n_{3},-n_{5}\right)$ \\
\hline$\omega \lambda 3$ & $\left(n_{5}, n_{6}\right) \rightarrow\left(n_{5}+n_{6}, n_{5}\right)$ & $\left(n_{3}, n_{4}, n_{5}, n_{6}\right) \rightarrow\left(n_{3}+n_{4}, n_{3}, n_{6}, n_{5}+n_{6}\right)$ \\
\hline$\theta \omega, \theta \lambda 2 \omega \lambda 5$ & $\left(n_{5}, n_{6}\right) \rightarrow\left(n_{5}+n_{6}, n_{5}\right)$ & $\left(n_{5}, n_{6}\right) \rightarrow\left(n_{6}, n_{5}+n_{6}\right)$ \\
\hline$\theta \omega \lambda 2, \theta \lambda 2 \omega \lambda 4$ & $I$ & $\left(n_{3}, n_{5}\right) \rightarrow\left(-n_{3},-n_{5}\right)$ \\
\hline$\theta \omega \lambda 3, \theta \lambda 2 \omega \lambda 3$ & $I$ & $\left(n_{3}, n_{4}\right) \rightarrow\left(n_{3}+n_{4}, n_{3}\right)$ \\
\hline$\theta \omega \lambda 4, \theta \lambda 2 \omega \lambda 2$ & $I$ & $n_{3} \rightarrow-n_{3}$ \\
\hline$\theta \omega \lambda 5, \theta \lambda 2 \omega$ & $I$ & $I$ \\
\hline
\end{tabular}


The moduli dependent suppression factors for the non-zero Yukawa couplings amongst twisted sectors containing massless states are given in the conventions of table 4 by

\begin{tabular}{|c|c|c|c|}
\hline $\begin{array}{c}\text { Yukawa } \\
\text { Coupling }\end{array}$ & $\lambda_{1}$ & $\lambda_{2}$ & $\lambda_{3}$ \\
\hline$T_{01} T_{13} T_{22}$ & 0 & $\mu\left(n_{3}, p_{3}, p_{4}\right)$ & 0 \\
\hline$T_{01} T_{14} T_{21}$ & 0 & 1 for $n_{3}\left(T_{14}\right) \neq 0$ & 0 \\
\hline$T_{02} T_{12} T_{22}$ & 0 & 2 for $m_{3}\left(T_{02}\right)-n_{3}\left(T_{12}\right) \neq 0$ & 1 for $n_{5}\left(T_{02}\right) \neq 0$ \\
\hline$T_{02} T_{13} T_{21}$ & 0 & $\mu\left(n_{3}, p_{3}, p_{4}\right)$ & 0 \\
\hline$T_{02} T_{14} T_{20}$ & 0 & 0 & 0 \\
\hline$T_{03} T_{11} T_{22}$ & 0 & $\mu\left(n_{3}, p_{3}, p_{4}\right)$ & $\mu\left(n_{5}, p_{5}, p_{6}\right)$ \\
\hline$T_{03} T_{12} T_{21}$ & 0 & $\mu\left(n_{3}, p_{3}, p_{4}\right)$ & $\mu\left(n_{5}, p_{5}, p_{6}\right)$ \\
\hline$T_{03} T_{13} T_{20}$ & 0 & 0 & 0 \\
\hline$T_{04} T_{10} T_{22}$ & 0 & 0 & $\mu\left(n_{5}, p_{5}, p_{6}\right)$ \\
\hline$T_{04} T_{11} T_{21}$ & 0 & 1 for $n_{3}\left(T_{04}\right) \neq 0$ & 1 for $n_{5}\left(T_{10}\right) \neq 0$ \\
\hline$T_{04} T_{12} T_{20}$ & 0 & 0 & $\mu\left(n_{5}, p_{5}, p_{6}\right)$ \\
\hline$T_{05} T_{10} T_{21}$ & 0 & 0 & 0 \\
\hline$T_{05} T_{11} T_{20}$ & 0 & 0 & 1 for $n_{5}\left(T_{10}\right) \neq 0$ \\
\hline$T_{10} T_{12} T_{14}$ & 2 for $m_{1}\left(T_{10}\right)-n_{1}\left(T_{12}\right) \neq 0$ & 0 & 0 \\
\hline$T_{10} T_{13} T_{13}$ & 2 for $m_{1}\left(T_{10}\right)-n_{1}\left(T_{13}\right) \neq 0$ & 0 & $m_{5}\left(T_{04}\right)-n_{5}\left(T_{12}\right) \neq 0$ \\
\hline$T_{11} T_{11} T_{14}$ & 2 for $m_{1}\left(T_{11}\right)-n_{1}\left(T_{11}\right) \neq 0$ & 1 for $n_{3}\left(T_{14}\right) \neq 0$ & $\left.p_{6}\right)$ \\
\hline$T_{11} T_{12} T_{13}$ & 2 for $m_{1}\left(T_{11}\right)-n_{1}\left(T_{12}\right) \neq 0$ & $\mu\left(n_{3}, p_{3}, p_{4}\right)$ & $m_{5}\left(T_{12}\right)-n_{5}\left(T_{12}\right) \neq 0$ \\
\hline$T_{12} T_{12} T_{12}$ & 2 for $m_{1}\left(T_{12}\right)-n_{1}\left(T_{12}\right) \neq 0$ & 2 for $m_{3}\left(T_{12}\right)-n_{3}\left(T_{12}\right) \neq 0$ & 2 for \\
\hline
\end{tabular}




\section{$\underline{\mathbf{Z}_{6} \times \mathbf{Z}_{6} \text { Orbifold }}$}

Lattice $G_{2} \lambda 3$

In the orthonormal basis,

$$
\begin{array}{ll}
\theta X_{1}=e \lambda 2 \pi i / 6 X_{1}, & \omega X_{1}=X_{1}, \\
\theta X_{2}=X_{2}, & \omega X_{2}=e \lambda 2 \pi i / 6 X_{2}, \\
\theta X_{3}=e \lambda-2 \pi i / 6 X_{3}, & \omega X_{3}=e \lambda-2 \pi i / 6 X_{3} .
\end{array}
$$

In the lattice basis,

$$
\theta=\left(C\left(G_{2}\right), I, C \lambda-1\left(G_{2}\right)\right), \quad \omega=\left(I, C\left(G_{2}\right), C \lambda-1\left(G_{2}\right)\right)
$$

so that

$$
\begin{aligned}
& \theta e_{1}=-e_{1}-e_{2}, \quad \theta e_{3}=e_{3}, \quad \theta e_{5}=2 e_{5}+e_{6}, \\
& \theta e_{2}=3 e_{1}+2 e_{2}, \quad \theta e_{4}=e_{4} \quad \theta e_{6}=-3 e_{5}-e_{6}
\end{aligned}
$$

and

$$
\begin{aligned}
& \omega e_{1}=e_{1}, \quad \omega e_{3}=-e_{3}-e_{4}, \quad \omega e_{5}=2 e_{5}+e_{6}, \\
& \omega e_{2}=e_{2}, \quad \omega e_{4}=3 e_{3}+2 e_{4}, \quad \omega e_{6}=-3 e_{5}-e_{6} .
\end{aligned}
$$

The independent deformation parameters (moduli) are $R_{1}, R_{3}$ and $R_{5}$, where

$$
\left|e_{2}\right|=\sqrt{3}\left|e_{1}\right| \equiv \sqrt{3} R_{1}, \quad\left|e_{4}\right|=\sqrt{3}\left|e_{3}\right| \equiv \sqrt{3} R_{3}, \quad\left|e_{6}\right|=\sqrt{3}\left|e_{5}\right| \equiv \sqrt{3} R_{5}
$$

also,

$$
\cos \theta_{12}=\cos \theta_{34}=\cos \theta_{56}=-\frac{\sqrt{3}}{2}
$$

with all other $\cos \theta_{i j}$ zero. A suitable choice of orthonormal basis $\tilde{e}_{1}, \ldots, \tilde{e}_{6}$ is given by

$$
\begin{aligned}
& e_{1}=R_{1} \tilde{e}_{1}, \quad e_{3}=R_{3} \tilde{e}_{3}, \quad e_{5}=R_{5} \tilde{e}_{5}, \\
& e_{2}=R_{1}\left(-\frac{3}{2} \tilde{e}_{1}-\frac{\sqrt{3}}{2} \tilde{e}_{2}\right), \quad e_{4}=R_{3}\left(-\frac{3}{2} \tilde{e}_{3}-\frac{\sqrt{3}}{2} \tilde{e}_{4}\right), \quad e_{6}=R_{5}\left(-\frac{3}{2} \tilde{e}_{5}-\frac{\sqrt{3}}{2} \tilde{e}_{6}\right) .
\end{aligned}
$$

The fixed points or tori and associated space group elements are given with the conventions of table 2 by 


\begin{tabular}{|c|c|c|}
\hline Twisted sector & Fixed point or torus & $l$ \\
\hline$\theta$ & $a_{3} e_{3}+a_{4} e_{4}$ & 0 \\
\hline$\theta \lambda 2$ & $-\frac{n_{1}}{3} e_{2}+\frac{n_{5}}{3} e_{6}+a_{3} e_{3}+a_{4} e_{4}$ & $n_{1} e_{1}+n_{5} e_{5}$ \\
\hline$\theta \lambda 3$ & $\frac{n_{1}}{2} e_{1}+\frac{n_{2}}{2} e_{2}+\frac{n_{5}}{2} e_{5}+\frac{n_{6}}{2} e_{6}+a_{3} e_{3}+a_{4} e_{4}$ & $n_{1} e_{1}+n_{2} e_{2}+n_{5} e_{5}+n_{6} e_{6}$ \\
\hline$\theta \lambda 4$ & $-\frac{n_{1}}{3} e_{2}+\frac{n_{5}}{3} e_{6}+a_{3} e_{3}+a_{4} e_{4}$ & $-n_{1} e_{1}-n_{5} e_{5}$ \\
\hline$\theta \lambda 5$ & $a_{3} e_{3}+a_{4} e_{4}$ & 0 \\
\hline$\omega$ & $a_{1} e_{1}+a_{2} e_{2}$ & 0 \\
\hline$\omega \lambda 2$ & $-\frac{n_{3}}{3} e_{4}+\frac{n_{5}}{3} e_{6}+a_{1} e_{1}+a_{2} e_{2}$ & $n_{3} e_{3}+n_{5} e_{5}$ \\
\hline$\omega \lambda 3$ & $\frac{n_{3}}{2} e_{3}+\frac{n_{4}}{2} e_{4}+\frac{n_{5}}{2} e_{5}+\frac{n_{6}}{2} e_{6}+a_{1} e_{1}+a_{2} e_{2}$ & $n_{3} e_{3}+n_{4} e_{4}+n_{5} e_{5}+n_{6} e_{6}$ \\
\hline$\omega \lambda 4$ & $-\frac{n_{3}}{3} e_{4}+\frac{n_{5}}{3} e_{6}+a_{1} e_{1}+a_{2} e_{2}$ & $-n_{3} e_{3}-n_{5} e_{5}$ \\
\hline$\omega \lambda 5$ & $a_{1} e_{1}+a_{2} e_{2}$ & 0 \\
\hline$\theta \omega$ & $\frac{n_{5}}{3} e_{6}$ & $n_{5} e_{5}$ \\
\hline$\theta \omega \lambda 2$ & $-\frac{n_{3}}{3} e_{4}+\frac{n_{5}}{2} e_{5}+\frac{n_{6}}{2} e_{6}$ & $n_{3} e_{3}+n_{5} e_{5}+n_{6} e_{6}$ \\
\hline$\theta \omega \lambda 3$ & $\frac{n_{3}}{2} e_{3}+\frac{n_{4}}{2} e_{4}+\frac{n_{5}}{3} e_{6}$ & $n_{3} e_{3}+n_{4} e_{4}-n_{5} e_{5}$ \\
\hline$\theta \omega \lambda 4$ & $-\frac{n_{3}}{3} e_{4}$ & $-n_{3} e_{3}$ \\
\hline$\theta \omega \lambda 5$ & $a_{5} e_{5}+a_{6} e_{6}$ & 0 \\
\hline$\theta \lambda 2 \omega$ & $-\frac{n_{1}}{3} e_{2}+\frac{n_{5}}{2} e_{5}+\frac{n_{6}}{2} e_{6}$ & $n_{1} e_{1}+n_{5} e_{5}+n_{6} e_{6}$ \\
\hline$\theta \lambda 2 \omega \lambda 2$ & $-\frac{n_{1}}{3} e_{2}-\frac{n_{3}}{3} e_{4}+\frac{n_{5}}{3} e_{6}$ & $n_{1} e_{1}+n_{3} e_{3}-n_{5} e_{5}$ \\
\hline$\theta \lambda 2 \omega \lambda 3$ & $-\frac{n_{1}}{3} e_{2}+\frac{n_{3}}{2} e_{3}+\frac{n_{4}}{2} e_{4}$ & $n_{1} e_{1}+n_{3} e_{3}+n_{4} e_{4}$ \\
\hline$\theta \lambda 2 \omega \lambda 4$ & $-\frac{n_{1}}{3} e_{2}-\frac{n_{3}}{3} e_{4}+a_{5} e_{5}+a_{6} e_{6}$ & $n_{1} e_{1}-n_{3} e_{3}$ \\
\hline$\theta \lambda 2 \omega \lambda 5$ & $-\frac{n_{1}}{3} e_{2}$ & $n_{1} e_{1}$ \\
\hline$\theta \lambda 3 \omega$ & $\frac{n_{1}}{2} e_{1}+\frac{n_{2}}{2} e_{2}+\frac{n_{5}}{3} e_{6}$ & $n_{1} e_{1}+n_{2} e_{2}-n_{5} e_{5}$ \\
\hline$\theta \lambda 3 \omega \lambda 2$ & $\frac{n_{1}}{2} e_{1}+\frac{n_{2}}{2} e_{2}-\frac{n_{3}}{3} e_{4}$ & $n_{1} e_{1}+n_{2} e_{2}+n_{3} e_{3}$ \\
\hline$\theta \lambda 3 \omega \lambda 3$ & $\frac{n_{1}}{2} e_{1}+\frac{n_{2}}{2} e_{2}+\frac{n_{3}}{2} e_{3}+\frac{n_{4}}{2} e_{4}+a_{5} e_{5}+a_{6} e_{6}$ & $n_{1} e_{1}+n_{2} e_{2}+n_{3} e_{3}+n_{4} e_{4}$ \\
\hline
\end{tabular}

Continued on next page 


\begin{tabular}{|c|c|c|}
\hline$\theta \lambda 3 \omega \lambda 4$ & $\frac{n_{1}}{2} e_{1}+\frac{n_{2}}{2} e_{2}-\frac{n_{3}}{3} e_{4}$ & $n_{1} e_{1}+n_{2} e_{2}-n_{3} e_{3}$ \\
\hline$\theta \lambda 3 \omega \lambda 5$ & $\frac{n_{1}}{2} e_{1}+\frac{n_{2}}{2} e_{2}+\frac{n_{5}}{2} e_{5}+\frac{n_{6}}{2} e_{6}$ & $n_{1} e_{1}+n_{2} e_{2}+n_{5} e_{5}+n_{6} e_{6}$ \\
\hline$\theta \lambda 4 \omega$ & $-\frac{n_{1}}{3} e_{2}$ & $-n_{1} e_{1}$ \\
\hline$\theta \lambda 4 \omega \lambda 2$ & $-\frac{n_{1}}{3} e_{2}-\frac{n_{3}}{3} e_{4}+a_{5} e_{5}+a_{6} e_{6}$ & $-n_{1} e_{1}+n_{3} e_{3}$ \\
\hline$\theta \lambda 4 \omega \lambda 3$ & $-\frac{n_{1}}{3} e_{2}+\frac{n_{3}}{2} e_{3}+\frac{n_{4}}{2} e_{4}$ & $-n_{1} e_{1}+n_{3} e_{3}+n_{4} e_{4}$ \\
\hline$\theta \lambda 4 \omega \lambda 4$ & $-\frac{n_{1}}{3} e_{2}-\frac{n_{3}}{3} e_{4}+\frac{n_{5}}{3} e_{6}$ & $-n_{1} e_{1}-n_{3} e_{3}+n_{5} e_{5}$ \\
\hline$\theta \lambda 4 \omega \lambda 5$ & $-\frac{n_{1}}{3} e_{2}+\frac{n_{5}}{2} e_{5}+\frac{n_{6}}{2} e_{6}$ & $-n_{1} e_{1}+n_{5} e_{5}+n_{6} e_{6}$ \\
\hline$\theta \lambda 5 \omega$ & $a_{5} e_{5}+a_{6} e_{6}$ & 0 \\
\hline$\theta \lambda 5 \omega \lambda 2$ & $-\frac{n_{3}}{3} e_{4}$ & $n_{3} e_{3}$ \\
\hline$\theta \lambda 5 \omega \lambda 3$ & $\frac{n_{3}}{2} e_{3}+\frac{n_{4}}{2} e_{4}+\frac{n_{5}}{3} e_{6}$ & $n_{3} e_{3}+n_{4} e_{4}+n_{5} e_{5}$ \\
\hline$\theta \lambda 5 \omega \lambda 4$ & $-\frac{n_{3}}{3} e_{4}+\frac{n_{5}}{2} e_{5}+\frac{n_{6}}{2} e_{6}$ & $-n_{3} e_{3}+n_{5} e_{5}+n_{6} e_{6}$ \\
\hline$\theta \lambda 5 \omega \lambda 5$ & $\frac{n_{5}}{3} e_{6}$ & $-n_{5} e_{5}$ \\
\hline
\end{tabular}


The action of $\theta$ and $\omega$ on the fixed points (or tori) is given by

\begin{tabular}{|c|c|c|}
\hline $\begin{array}{l}\text { twisted } \\
\text { sector }\end{array}$ & Action of $\theta$ & Action of $\omega$ \\
\hline$\theta, \theta \lambda 5$ & $I$ & $I$ \\
\hline$\theta \lambda 2, \theta \lambda 4$ & $\left(n_{1}, n_{5}\right) \rightarrow\left(-n_{1},-n_{5}\right)$ & $n_{5} \rightarrow-n_{5}$ \\
\hline$\theta \lambda 3$ & $\left(n_{1}, n_{2}, n_{5}, n_{6}\right) \rightarrow\left(n_{1}+n_{2}, n_{1}, n_{6}, n_{5}+n_{6}\right)$ & $\left(n_{5}, n_{6}\right) \rightarrow\left(n_{6}, n_{5}+n_{6}\right)$ \\
\hline$\omega, \omega \lambda 5$ & $I$ & $I$ \\
\hline$\omega \lambda 2, \omega \lambda 4$ & $n_{5} \rightarrow-n_{5}$ & $\left(n_{3}, n_{5}\right) \rightarrow\left(-n_{3},-n_{5}\right)$ \\
\hline$\omega \lambda 3$ & $\left(n_{5}, n_{6}\right) \rightarrow\left(n_{6}, n_{5}+n_{6}\right)$ & $\left(n_{3}, n_{4}, n_{5}, n_{6}\right) \rightarrow\left(n_{3}+n_{4}, n_{3}, n_{6}, n_{5}+n_{6}\right.$ \\
\hline$\theta \omega, \theta \lambda 5 \omega \lambda 5$ & $n_{5} \rightarrow-n_{5}$ & $n_{5} \rightarrow-n_{5}$ \\
\hline$\theta \omega \lambda 2, \theta \lambda 5 \omega \lambda 4$ & $\left(n_{5}, n_{6}\right) \rightarrow\left(n_{6}, n_{5}+n_{6}\right)$ & $\left(n_{3}, n_{5}, n_{6}\right) \rightarrow\left(-n_{3}, n_{6}, n_{5}+n_{6}\right)$ \\
\hline$\theta \omega \lambda 3, \theta \lambda 5 \omega \lambda 3$ & $n_{5} \rightarrow-n_{5}$ & $\left(n_{3}, n_{4}, n_{5}\right) \rightarrow\left(n_{3}+n_{4}, n_{3},-n_{5}\right)$ \\
\hline$\theta \omega \lambda 4, \theta \lambda 5 \omega \lambda 2$ & $I$ & $n_{3} \rightarrow-n_{3}$ \\
\hline$\theta \omega \lambda 5, \theta \lambda 5 \omega$ & $I$ & $I$ \\
\hline$\theta \lambda 2 \omega, \theta \lambda 4 \omega \lambda 5$ & $\left(n_{1}, n_{5}, n_{6}\right) \rightarrow\left(-n_{1}, n_{6}, n_{5}+n_{6}\right)$ & $\left(n_{5}, n_{6}\right) \rightarrow \rightarrow\left(n_{6}, n_{5}+n_{6}\right)$ \\
\hline$\theta \lambda 2 \omega \lambda 2, \theta \lambda 4 \omega \lambda 4$ & $\left(n_{1}, n_{5}\right) \rightarrow\left(-n_{1},-n_{5}\right)$ & $\left(n_{3}, n_{5}\right) \rightarrow\left(-n_{3},-n_{5}\right)$ \\
\hline$\theta \lambda 2 \omega \lambda 3, \theta \lambda 4 \omega \lambda 3$ & $n_{1} \rightarrow-n_{1}$ & $\left(n_{3}, n_{4}\right) \rightarrow\left(n_{3}+n_{4}, n_{3}\right)$ \\
\hline$\theta \lambda 2 \omega \lambda 4, \theta \lambda 4 \omega \lambda 2$ & $n_{1} \rightarrow-n_{1}$ & $n_{3} \rightarrow-n_{3}$ \\
\hline$\theta \lambda 2 \omega \lambda 5, \theta \lambda 4 \omega$ & $n_{1} \rightarrow-n_{1}$ & $I$ \\
\hline$\theta \lambda 3 \omega, \theta \lambda 3 \omega \lambda 5$ & $\left(n_{1}, n_{2}, n_{5}\right) \rightarrow\left(n_{1}+n_{2}, n_{1},-n_{5}\right)$ & $n_{5} \rightarrow-n_{5}$ \\
\hline$\theta \lambda 3 \omega \lambda 2, \theta \lambda 3 \omega \lambda 4$ & $\left(n_{1}, n_{2}\right) \rightarrow\left(n_{1}+n_{2}, n_{1}\right)$ & $n_{3} \rightarrow-n_{3}$ \\
\hline$\theta \lambda 3 \omega \lambda 3$ & $\left(n_{1}, n_{2}\right) \rightarrow\left(n_{1}+n_{2}, n_{1}\right)$ & $\left(n_{3}, n_{4}\right) \rightarrow\left(n_{3}+n_{4}, n_{3}\right)$ \\
\hline
\end{tabular}

The moduli dependent suppression factors for the non-zero Yukawa couplings amongst twisted sectors containing massless states are given in the conventions of table 4 by 


\begin{tabular}{|c|c|c|c|}
\hline Yukawa Coupling & $\lambda_{1}$ & $\lambda_{2}$ & $\lambda_{3}$ \\
\hline$T_{01} T_{14} T_{51}$ & 0 & 1 for $n_{3}\left(T_{14}\right) \neq 0$ & 0 \\
\hline$T_{01} T_{15} T_{50}$ & 0 & 0 & 0 \\
\hline$T_{01} T_{23} T_{42}$ & 0 & $\mu\left(n_{3}, p_{3}, p_{4}\right)$ & 0 \\
\hline$T_{01} T_{24} T_{41}$ & 0 & 1 for $n_{3}\left(T_{24}\right) \neq 0$ & 0 \\
\hline$T_{01} T_{32} T_{33}$ & 0 & $\mu\left(n_{3}, p_{3}, p_{4}\right)$ & 0 \\
\hline$T_{02} T_{13} T_{51}$ & 0 & $\mu\left(n_{3}, p_{3}, p_{4}\right)$ & 0 \\
\hline$T_{02} T_{14} T_{50}$ & 0 & 0 & 1 for $n_{5}\left(T_{02}\right) \neq 0$ \\
\hline$T_{02} T_{22} T_{42}$ & 0 & 2 for $m_{3}\left(T_{02}\right)-n_{3}\left(T_{22}\right) \neq 0$ & 0 \\
\hline$T_{02} T_{23} T_{41}$ & 0 & $\mu\left(n_{3}, p_{3}, p_{4}\right)$ & 1 for $n_{5}\left(T_{02}\right) \neq 0$ \\
\hline$T_{02} T_{24} T_{40}$ & 0 & 0 & 0 \\
\hline$T_{02} T_{31} T_{33}$ & 0 & $\mu\left(n_{3}, p_{3}, p_{4}\right)$ & 0 \\
\hline$T_{02} T_{32} T_{32}$ & 0 & 2 for $m_{3}\left(T_{02}\right)-n_{3}\left(T_{32}\right) \neq 0$ & 1 for $n_{5}\left(T_{02}\right) \neq 0$ \\
\hline$T_{03} T_{12} T_{51}$ & 0 & $\mu\left(n_{3}, p_{3}, p_{4}\right)$ & 0 \\
\hline$T_{03} T_{13} T_{50}$ & 0 & 0 & $\mu\left(n_{5}, p_{5}, p_{6}\right)$ \\
\hline$T_{03} T_{21} T_{42}$ & 0 & $\mu\left(n_{3}, p_{3}, p_{4}\right)$ & 0 \\
\hline$T_{03} T_{22} T_{41}$ & 0 & $\mu\left(n_{3}, p_{3}, p_{4}\right)$ & $\mu\left(n_{5}, p_{5}, p_{6}\right)$ \\
\hline$T_{03} T_{23} T_{40}$ & 0 & 0 & $\mu\left(n_{5}, p_{5}, p_{6}\right)$ \\
\hline$T_{03} T_{30} T_{33}$ & 0 & 0 & 0 \\
\hline$T_{03} T_{31} T_{32}$ & 0 & $\mu\left(n_{3}, p_{3}, p_{4}\right)$ & $\mu\left(n_{5}, p_{5}, p_{6}\right)$ \\
\hline$T_{04} T_{11} T_{51}$ & 0 & 1 for $n_{3}\left(T_{04}\right) \neq 0$ & 0 \\
\hline$T_{04} T_{12} T_{50}$ & 0 & 0 & $\mu\left(n_{5}, p_{5}, p_{6}\right)$ \\
\hline$T_{04} T_{20} T_{42}$ & 0 & 0 & 0 \\
\hline$T_{04} T_{21} T_{41}$ & 0 & 1 for $n_{3}\left(T_{04}\right) \neq 0$ & $\mu\left(n_{5}, p_{5}, p_{6}\right)$ \\
\hline$T_{04} T_{22} T_{40}$ & 0 & 0 & 2 for $n_{5}\left(T_{04}\right)-n_{5}\left(T_{22}\right) \neq 0$ \\
\hline
\end{tabular}

Continued on next page 


\begin{tabular}{|c|c|c|c|}
\hline$T_{04} T_{30} T_{32}$ & 0 & 0 & $\mu\left(n_{5}, p_{5}, p_{6}\right)$ \\
\hline$T_{05} T_{10} T_{51}$ & 0 & 0 & 0 \\
\hline$T_{05} T_{11} T_{50}$ & 0 & 0 & 1 for $n_{5}\left(T_{11}\right) \neq 0$ \\
\hline$T_{05} T_{20} T_{41}$ & 0 & 0 & 1 for $n_{5}\left(T_{20}\right) \neq 0$ \\
\hline$T_{05} T_{21} T_{40}$ & 0 & 0 & $\mu\left(n_{5}, p_{5}, p_{6}\right)$ \\
\hline$T_{05} T_{30} T_{31}$ & 0 & 0 & $\mu\left(n_{5}, p_{5}, p_{6}\right)$ \\
\hline$T_{10} T_{14} T_{42}$ & 1 for $n_{1}\left(T_{42}\right) \neq 0$ & 0 & 0 \\
\hline$T_{10} T_{15} T_{41}$ & 1 for $n_{1}\left(T_{41}\right) \neq 0$ & 0 & 0 \\
\hline$T_{10} T_{23} T_{33}$ & $\mu\left(n_{1}, p_{1}, p_{2}\right)$ & 0 & 0 \\
\hline$T_{10} T_{24} T_{32}$ & $\mu\left(n_{1}, p_{1}, p_{2}\right)$ & 0 & 0 \\
\hline$T_{11} T_{13} T_{42}$ & 1 for $n_{1}\left(T_{42}\right) \neq 0$ & $\mu\left(n_{3}, p_{3}, p_{4}\right)$ & 0 \\
\hline$T_{11} T_{14} T_{41}$ & 1 for $n_{1}\left(T_{41}\right) \neq 0$ & 1 for $n_{3}\left(T_{14}\right) \neq 0$ & 1 for $n_{5}\left(T_{11}\right) \neq 0$ \\
\hline$T_{11} T_{15} T_{40}$ & 1 for $n_{1}\left(T_{40}\right) \neq 0$ & 0 & 0 \\
\hline$T_{11} T_{22} T_{33}$ & $\mu\left(n_{1}, p_{1}, p_{2}\right)$ & $\mu\left(n_{3}, p_{3}, p_{4}\right)$ & 0 \\
\hline$T_{11} T_{23} T_{32}$ & $\mu\left(n_{1}, p_{1}, p_{2}\right)$ & $\mu\left(n_{3}, p_{3}, p_{4}\right)$ & 1 for $n_{5}\left(T_{11}\right) \neq 0$ \\
\hline$T_{11} T_{24} T_{31}$ & $\mu\left(n_{1}, p_{1}, p_{2}\right)$ & 1 for $n_{3}\left(T_{24}\right) \neq 0$ & 0 \\
\hline$T_{12} T_{12} T_{42}$ & 1 for $n_{1}\left(T_{42}\right) \neq 0$ & 2 for $m_{3}\left(T_{12}\right)-n_{3}\left(T_{12}\right) \neq 0$ & 0 \\
\hline$T_{12} T_{13} T_{41}$ & 1 for $n_{1}\left(T_{41}\right) \neq 0$ & $\mu\left(n_{3}, p_{3}, p_{4}\right)$ & $\mu\left(n_{5}, p_{5}, p_{6}\right)$ \\
\hline$T_{12} T_{14} T_{40}$ & 1 for $n_{1}\left(T_{40}\right) \neq 0$ & 0 & $\mu\left(n_{5}, p_{5}, p_{6}\right)$ \\
\hline$T_{12} T_{21} T_{33}$ & $\mu\left(n_{1}, p_{1}, p_{2}\right)$ & $\mu\left(n_{3}, p_{3}, p_{4}\right)$ & 0 \\
\hline$T_{12} T_{22} T_{32}$ & $\mu\left(n_{1}, p_{2}, p_{2}\right)$ & 2 for $m_{3}\left(T_{12}\right)-n_{3}\left(T_{22}\right) \neq 0$ & $\mu\left(n_{5}, p_{5}, p_{6}\right)$ \\
\hline$T_{12} T_{23} T_{31}$ & $\mu\left(n_{1}, p_{1}, p_{2}\right)$ & $\mu\left(n_{3}, p_{3}, p_{4}\right)$ & $\mu\left(n_{5}, p_{5}, p_{6}\right)$ \\
\hline$T_{12} T_{24} T_{30}$ & $\mu\left(n_{1}, p_{1}, p_{2}\right)$ & 0 & 0 \\
\hline$T_{13} T_{13} T_{40}$ & 1 for $n_{1}\left(T_{40}\right) \neq 0$ & 0 & 2 for $m_{5}\left(T_{13}\right)-n_{5}\left(T_{13}\right) \neq 0$ \\
\hline$T_{13} T_{20} T_{33}$ & $\mu\left(n_{1}, p_{1}, p_{2}\right)$ & 0 & 0 \\
\hline
\end{tabular}

Continued on next page 


\begin{tabular}{|l|c|c|c|}
\hline$T_{13} T_{21} T_{32}$ & $\mu\left(n_{1}, p_{1}, p_{2}\right)$ & $\mu\left(n_{3}, p_{3}, p_{4}\right)$ & $\mu\left(n_{5}, p_{5}, p_{6}\right)$ \\
\hline$T_{13} T_{22} T_{31}$ & $\mu\left(n_{1}, p_{1}, p_{2}\right)$ & $\mu\left(n_{3}, p_{3}, p_{4}\right)$ & 2 for $m_{5}\left(T_{22}\right)-n_{5}\left(T_{13}\right) \neq 0$ \\
\hline$T_{13} T_{23} T_{30}$ & $\mu\left(n_{1}, p_{1}, p_{2}\right)$ & 0 & $\mu\left(n_{5}, p_{5}, p_{6}\right)$ \\
\hline$T_{14} T_{20} T_{32}$ & $\mu\left(n_{1}, p_{1}, p_{2}\right)$ & 0 & 1 for $n_{5}\left(T_{20}\right) \neq 0$ \\
\hline$T_{14} T_{21} T_{31}$ & $\mu\left(n_{1}, p_{1}, p_{2}\right)$ & 1 for $n_{3}\left(T_{14}\right) \neq 0$ & $\mu\left(n_{5}, p_{5}, p_{6}\right)$ \\
\hline$T_{14} T_{22} T_{30}$ & $\mu\left(n_{1}, p_{1}, p_{2}\right)$ & 0 & $\mu\left(n_{5}, p_{5}, p_{6}\right)$ \\
\hline$T_{15} T_{20} T_{31}$ & $\mu\left(n_{1}, p_{1}, p_{2}\right)$ & 0 & 0 \\
\hline$T_{15} T_{21} T_{30}$ & $\mu\left(n_{1}, p_{1}, p_{2}\right)$ & 0 & 0 \\
\hline$T_{20} T_{22} T_{24}$ & 2 for $m_{1}\left(T_{20}\right)-n_{1}\left(T_{22}\right) \neq 0$ & 0 & 0 \\
\hline$T_{20} T_{23} T_{23}$ & 2 for $m_{1}\left(T_{20}\right)-n_{1}\left(T_{23}\right) \neq 0$ & 1 for $n_{3}\left(T_{20}\right) \neq 0$ & 0 \\
\hline$T_{21} T_{21} T_{24}$ & 2 for $m_{1}\left(T_{21}\right)-n_{1}\left(T_{21}\right) \neq 0$ & 1 for $n_{3}\left(T_{24}\right) \neq 0$ & 0 \\
\hline$T_{21} T_{22} T_{23}$ & 2 for $m_{1}\left(T_{21}\right)-n_{1}\left(T_{22}\right) \neq 0$ & $\mu\left(n_{3}, p_{3}, p_{4}\right)$ & $\mu\left(n_{5}, p_{5}, p_{6}\right)$ \\
\hline$T_{22} T_{22} T_{22}$ & 2 for $m_{1}\left(T_{22}\right)-n_{1}\left(T_{22}\right) \neq 0$ & 2 for $m_{3}\left(T_{22}\right)-n_{3}\left(T_{22}\right) \neq 0$ & 2 for $m_{5}\left(T_{22}\right)-n_{5}\left(T_{22}\right) \neq 0$ \\
\hline
\end{tabular}

